TRANSACTIONS OF THE

AMERICAN MATHEMATICAL SOCIETY

Volume 362, Number 4, April 2010, Pages 1907-1936

S 0002-9947(09)04752-7

Article electronically published on November 13, 2009

\title{
EXISTENCE AND NON-EXISTENCE RESULTS FOR A LOGISTIC-TYPE EQUATION ON MANIFOLDS
}

\author{
STEFANO PIGOLA, MARCO RIGOLI, AND ALBERTO G. SETTI
}

Dedicated to the memory of Franca Burrone Rigoli

\begin{abstract}
We study the steady state solutions of a generalized logistic-type equation on a complete Riemannian manifold. We provide sufficient conditions for existence, respectively non-existence of positive solutions, which depend on the relative size of the coefficients and their mutual interaction with the geometry of the manifold, which is mostly taken into account by means of conditions on the volume growth of geodesic balls.
\end{abstract}

\section{INTRODUCTION}

The aim of this paper is to study the problem of existence, non-existence and uniqueness of solutions of the equation

$$
\Delta u+a(x) u-b(x) u^{\sigma}=0 \quad \text { on } M,
$$

on a complete, connected Riemannian manifold $(M,\langle\rangle$,$) . Here \sigma>1$, the coefficient $b(x)$ is assumed to be non-negative while $a(x)$ is not assumed to be of constant sign.

Equations of the form (0.1) arise in Riemannian geometry, as the equation for the change of the scalar curvature under a conformal change of the metric (see, e.g., $[\mathrm{K}]$ ) and in mathematical biology, where they describe the steady state solutions of the logistic equation with diffusion

$$
\frac{\partial}{\partial t} u=\Delta u+a(x) u-b(x) u^{\sigma}
$$

(see, e.g., [AB], $\mathrm{AW}$, [DM1, [DM2]). In the latter context $u$ represents the density of a population, and it is therefore assumed to be non-negative, the non-linear term $-b(x) u^{\sigma}$ accounts for the fact that the population is self-limiting, and the function $a(x)$ represents the birth rate of the population, with no self-limitation.

In the Euclidean setting, G.A. Afrouzi and K.J Brown, $\mathrm{AB}$, have studied the following special case of (0.1):

$$
\Delta u+\lambda\left[g(x) u-u^{2}\right]=0 \quad \text { on } \mathbb{R}^{m},
$$

where the positive parameter $\lambda>0$ is the inverse of the diffusion rate, and $g(x)$ is a changing sign coefficient which again represents the birth rate of the population.

Received by the editors February 17, 2006 and, in revised form, September 7, 2007.

2000 Mathematics Subject Classification. Primary 58J05, 58J50; Secondary 35J60, 35P05, $53 \mathrm{C} 21$.

Key words and phrases. Nonlinear elliptic equations, existence and non-existence results, eigenvalues, Riemannian manifolds. 
Their results describe the interplay between diffusion, and birth rate, and show that if the diffusion is sufficiently small, solutions may exist even if $a$ is predominantly negative, while if the diffusion is large, then solutions exist only if the birth rate is sufficiently large.

In fact, the mutual interactions between diffusion and birth rate are often taken into account by the principal eigenvalue $\lambda_{*}$ of the linear part of equation (0.3) (see Section 1 below for the relevant definitions). This is exemplified, e.g., in $\mathrm{AB}$, Theorem 2.2, where it is proved that if $g$ is positive somewhere, so that $\lambda_{*} \geq 0$, and if $\lambda>\lambda_{*}$, then equation (0.3) has a positive solution.

Further (see Section 3 therein), under the additional assumption that $g(x)$ is strictly negative in the complement of a ball, (0.3) has (exactly one) positive solution if $\lambda>\lambda_{*}$, and no positive solution if $\lambda \leq \lambda_{*}$.

More recently, existence, non-existence, and uniqueness results have been obtained by Y. Du and L. Ma, DM2], who study the equation

$$
\Delta u+\lambda g(x) u-b(x) u^{\sigma}=0 \quad \text { on } \mathbb{R}^{m},
$$

where $\sigma>1$ and $b(x)$ is a non-negative coefficient. They show that if $g$ is positive somewhere and its positive part satisfies suitable conditions, which in particular imply that $\lambda_{*}>0$, and if $b$ is strictly positive outside a bounded connected open set $S_{o}$ with principal eigenvalue $\lambda_{1}\left(S_{o}\right)\left(=+\infty\right.$ if $S_{o}$ is empty), then (0.4) has a (unique) solution in the homogeneous Sobolev space $H_{h}^{1}\left(\mathbb{R}^{m}\right)$ if $\lambda_{*}<\lambda<\lambda_{1}\left(S_{o}\right)$, no solution in $H_{h}^{1}\left(\mathbb{R}^{m}\right)$ if $0<\lambda \leq \lambda_{*}$ and no solutions at all if $\lambda_{1}\left(S_{o}\right)<+\infty$ and $\lambda \geq \lambda_{1}\left(S_{o}\right)$.

Our results provide new insight on the interplay between diffusion and growth rate, that is, in our notation, between the relative size of the variable coefficients $a(x)$ and $b(x)$. Indeed, we show that if $a(x)$ is sufficiently large in a suitable ball, while outside the ball the negative part of $a(x)$ is not too large (so a possibly overall negative birth rate is compensated by a sufficiently large positive birth rate in the ball), then (0.1) has a positive solution (see Theorem 2.10 below), independently of the size of $b(x)$. On the other hand, the content of Theorem 3.6 is that if $a(x)$ is sufficiently small compared to $b(x)$, and certain geometric conditions on the volume growth of the manifold hold, then (0.1) has no positive solution. We note that in the special case where $b$ is constant, and the underlying manifold is Euclidean space, Theorem 3.6 generalizes and complements the non-existence result contained in $\mathrm{AB}$, Section 3.

Observe also that, according to Theorem 2.1 in BRS2, if $a(x)$ is sufficiently large that the bottom of the spectrum $\lambda_{1}^{\Delta+a(x)}(M)$ of the Schrödinger operator $\Delta+a(x)$ is negative, then one can guarantee the existence of a (minimal) solution of (0.1) irrespectively of the size of $b(x)$. This is tightly related to the abovementioned relationship between the existence of steady state solutions and the principal eigenvalue $\lambda_{*}$ of the problem $\Delta u+\lambda a(x) u=0$ on $M$. Indeed, as we shall explain in Section $1, \lambda_{*}$ is precisely the largest value of $\lambda$ for which $\lambda_{1}^{\Delta+\lambda a(x)}(M) \geq$ 0 .

Note however, that in our main existence result, Theorem 2.10, we avoid an assumption of this type and describe explicit conditions on the coefficients that guarantee existence, thus giving a new contribution to the subject.

It should also be stressed that having replaced Euclidean space with a Riemannian manifold, the behavior of the equation is now sensitive to the geometry of the 
underlying space, and therefore reflects not only the mutual relationship of the coefficients $a(x)$ and $b(x)$, but also their respective interaction with the geometry. From the analytic point of view this introduces new difficulties. For instance, to prove our main non-existence result we need to determine an asymptotic a priori upper bound for the solution $u$, and the techniques that are usually employed in the Euclidean setting are not available. We overcome the problem via a different approach of geometric flavor, which may be of independent interest (see Lemma 3.5).

From now on we denote by $(M,\langle\rangle$,$) a connected, complete, non-compact Rie-$ mannian manifold of dimension $m \geq 2$. We fix a reference point $o$ in $M$, and denote by $r(x)$ the Riemannian distance function from $o$, and by $B_{R}$ and $\partial B_{R}$ the geodesic ball and sphere, respectively, of radius $R>0$ centered at $o$. Finally, we will denote by $C$, possibly with subscripts or superscripts, a positive constant which may vary from place to place and that may depend on any factor quantified (implicitly or explicitly) before its occurrence, but not on factors quantified afterwards. Given functions $A$ and $B$, defined on a set $\Omega$, we say that $A=O(B)$ in $\Omega$ if there exists $C$ such that

$$
A(t) \leq C B(t) \quad \forall t \in \Omega .
$$

\section{On the PRincipal Eigenvalue $\lambda_{*}$}

As mentioned in the Introduction, existence results for equation (0.3) typically depend on the assumption that the parameter $\lambda$ be strictly greater than the principal eigenvalue of the linear part of the equation.

Recall that a constant $\lambda_{1}$ is said to be a principal eigenvalue for the linear equation

$$
\Delta u+\lambda a(x) u=0
$$

if for $\lambda=\lambda_{1}$ the equation has a positive solution.

On the other hand, in the literature on the non-compact Yamabe equation, existence results often depend on the assumption that the sign of the bottom of the spectrum of the Schrödinger operator associated to the equation be negative (see, e.g., [BRS2]).

It is therefore natural to investigate the relationships between principal and spectral eigenvalues.

Let $a(x) \in C^{\infty}(M)$ and, given a fixed radius $R$, consider the eigenvalue problem

$$
\begin{cases}\Delta \varphi+\lambda a(x) \varphi=0 & \text { on } B_{R}, \lambda \in \mathbb{R}, \\ \varphi=0 & \text { on } \partial B_{R} .\end{cases}
$$

If $a\left(x_{o}\right)>0$ for some $x_{o} \in B_{R}$, then it is well known (see [MM], [HK]) that (1.1) has a positive principal eigenvalue $\lambda_{1}\left(B_{R}\right)$, which is variationally characterized by

$$
\lambda_{1}(R)=\inf \left\{\int_{B_{R}}|\nabla u|^{2}: u \in H_{o}^{1}\left(B_{R}\right), \int_{B_{R}} a(x) u^{2}=1\right\},
$$

and a principal positive eigenfunction $\varphi$ on $B_{R}$ satisfying

$$
\begin{cases}\Delta \varphi+\lambda_{1}(R) a(x) \varphi=0 & \text { on } B_{R} \\ \varphi=0 & \text { on } \partial B_{R} .\end{cases}
$$

We note in passing that, by the maximum principle, the condition that $a(x)$ is positive somewhere in $B_{R}$ is also necessary for the existence of a positive principal eigenvalue. 
It follows from (1.2) that $\lambda_{1}(R)$ is a non-increasing function of $R$, and we may set

$$
\lambda_{*}=\lim _{R \rightarrow+\infty} \lambda_{1}(R) \geq 0 .
$$

On the other hand, for $\mu \in \mathbb{R}$, let $L_{\mu}$ be the operator $L_{\mu}=\Delta+\mu a(x)$ and denote by $\lambda_{1}\left(L_{\mu}, R\right)$ the first Dirichlet eigenvalue of $L_{\mu}$ on $B_{R}$, so that

$$
\lambda_{1}\left(L_{\mu}, R\right)=\inf \left\{\int_{B_{R}}|\nabla u|^{2}-\mu a(x) u^{2}: u \in H_{o}^{1}\left(B_{R}\right), \int_{B_{R}} u^{2}=1\right\},
$$

and there exists a smooth positive eigenfunction $\psi$ of $L_{\mu}$ on $B_{R}$ satisfying

$$
\begin{cases}L_{\mu} \psi=-\lambda_{1}\left(L_{\mu}, R\right) \psi & \text { on } B_{R} \\ \psi=0 & \text { on } \partial B_{R}\end{cases}
$$

Again $\lambda_{1}\left(L_{\mu}, R\right)$ is a non-increasing function of $R$ and one may define

$$
\lambda_{1}^{L_{\mu}}(M)=\lim _{R \rightarrow+\infty} \lambda_{1}\left(L_{\mu}, R\right),
$$

which coincides with the bottom of the $L^{2}$-spectrum of $L_{\mu}$ in the case where the operator is essentially selfadjoint on $C_{c}^{\infty}(M)$ (this happens, e.g., if the operator $L_{\mu}$ is bounded from below on $C_{c}^{\infty}$; see $\mathrm{BdCS}$, Proposition 2).

By a result of W.F. Moss and J. Pieperbrink, $\mathrm{MP}$, and D. Fischer-Colbrie and R. Schoen, [FCS], we have that $\lambda_{1}^{L_{\mu}}(M) \geq 0$ if and only if there exists a positive solution $u \in C^{\infty}(M)$ of

$$
\Delta u+\mu a(x) u=0
$$

on $M$.

We are now ready to prove the following.

Proposition 1.1. Let $a(x) \in C^{\infty}(M)$ satisfy $a\left(x_{o}\right)>0$ for some $x_{o} \in M$. Then

$$
\lambda_{*}=\sup \left\{\mu \geq 0: \lambda_{1}^{L_{\mu}}(M) \geq 0\right\} .
$$

Proof. Fix $R_{o}$ sufficiently large that $x_{o} \in B_{R_{o}}$, and choose a sequence $R_{k}$ such that $R_{o}<R_{k} \nearrow+\infty$. Denote by $\varphi_{k}$ the solution of (1.3) on $B_{R_{k}}$ with principal eigenvalue $\lambda_{1}\left(R_{k}\right)$, normalized with $\varphi_{k}\left(x_{o}\right)=1$. Arguing as in the proof of [J], Theorem 1, one shows that $\left\{\varphi_{k}\right\}$ has a subsequence which converges locally uniformly on $M$ to a $C^{\infty}$ non-negative function $\varphi$ satisfying $\varphi\left(x_{o}\right)=1$ and

$$
\Delta \varphi+\lambda_{*} a(x) \varphi=0 \quad \text { on } M
$$

Furthermore, by the maximum principle (see [GT], p. 35), $\varphi>0$ on $M$. It follows from (1.6) that $\lambda_{1}^{L_{\lambda *}}(M) \geq 0$, so that

$$
\lambda_{*} \leq \sup \left\{\mu \geq 0: \lambda_{1}^{L_{\mu}}(M) \geq 0\right\} .
$$

On the other hand, let $\mu \geq 0$ be such that $\lambda_{1}^{L_{\mu}}(M) \geq 0$. We claim that $\mu \leq \lambda_{*}$ so that the reverse inequality holds in the above formula, and the required conclusion follows. To this end, let $u$ be a smooth positive function satisfying (1.6), and fix $R>0$ sufficiently large that $x_{o} \in B_{R}$. Defining $w=\log u$, it follows from (1.6) that

$$
\Delta w=-\mu a(x)-|\nabla w|^{2} .
$$


Given any $v \in C_{0}^{\infty}\left(B_{R}\right), v \not \equiv 0$, we multiply both sides of (1.7) times $v^{2}$, integrate by parts and use Young's inequality to obtain

$$
\int_{B_{R}} \mu a(x) v^{2}+|\nabla w|^{2} v^{2}=\int_{B_{R}} 2 v\langle\nabla v, \nabla w\rangle \leq \int_{B_{R}}|\nabla w|^{2} v^{2}+|\nabla v|^{2},
$$

whence

$$
\mu \int_{B_{R}} a(x) v^{2} \leq \int_{B_{R}}|\nabla v|^{2}
$$

Now the variational characterization of the principal eigenvalue shows that $\mu \leq$ $\lambda_{1}(R)$ and the claim follows from the definition of $\lambda_{*}$.

Corollary 1.2. Let $a(x) \in C^{\infty}(M)$ be such that $a\left(x_{o}\right)>0$ for some $x_{o} \in M$. Then a non-negative number $\mu$ satisfies $\mu>\lambda_{*}$ if and only if $\lambda_{1}^{L_{\mu}}(M)<0$.

Proof. Since in our assumptions $\lambda_{*} \geq 0$, we may assume that $\mu>0$. Assume by contradiction that $\lambda_{1}^{L_{\mu}}(M)<0$ and $\mu \leq \lambda_{*}$. By definition, there exists $R$ sufficiently large that $x_{o} \in B_{R}$ and $\lambda_{1}\left(L_{\mu}, R\right)<0$, so that, if $\psi$ is the corresponding positive eigenfunction as in (1.5), we have

$$
\int_{B_{R}}|\nabla \psi|^{2} \leq \mu \int_{B_{R}} a(x) \psi^{2}
$$

In particular, the integral on the right hand side is positive, and since $\psi \in H_{0}^{1}\left(B_{R}\right)$, we have

$$
\lambda_{*} \leq \lambda_{1}(R) \leq \frac{\int_{B_{R}}|\nabla \psi|^{2}}{\int_{B_{R}} a(x) \psi^{2}}<\mu,
$$

which gives the required contradiction. The reverse implication is an immediate consequence of Proposition 1.1 .

We remark that statements similar to Proposition 1.1 and Corollary 1.2 hold (almost trivially) in the case of a bounded domain $\Omega$ with smooth boundary such that $a\left(x_{o}\right)>0$ for some $x_{o} \in \Omega$.

We conclude this section by showing an application of the results obtained to the case of the Schrödinger operator $\Delta+\lambda a(x)$ on $\mathbb{R}^{m}$. We assume that the positive part $a_{+}(x)$ of $a(x)$ does not vanish identically, so that the results described above hold, and that it satisfies the estimate

$$
a_{+}(x) \leq \frac{k}{|x|^{2}}
$$

for some positive constant $k$. According to BRS1], Lemma 2.3, if $A(t) \leq \frac{(m-2)^{2}}{4 t^{2}}$, then the equation

$$
\Delta \varphi+A(|x|) \varphi=0
$$

has a positive solution $\varphi$ on $\mathbb{R}^{m}$. Thus, if $\lambda k \leq(m-2)^{2} / 4$, then $\varphi$ satisfies

$$
\Delta \varphi+\lambda a(x) \varphi \leq 0
$$

which according to the above-mentioned result of Fischer-Colbrie and Schoen, FCS, gives $\lambda_{1}^{\Delta+\lambda a(x)}(M) \geq 0$. We conclude that $\lambda_{*}$ is strictly positive, and, in fact,

$$
\lambda_{*} \geq \frac{(m-2)^{2}}{4 k} .
$$

We will come back to this in Section 3 below. 


\section{EXISTENCE AND UNiqUENESS RESUlts}

The established relationship between $\lambda_{*}$ and $\lambda_{1}^{L_{\mu}}(M)$ allows us to apply to the present situation many of the results obtained in BRS2. In particular, we quote the following theorem, which states the existence of minimal positive solutions of equation (0.1).

Theorem 2.1. Let $a(x), b(x) \in C_{l o c}^{0, \alpha}(M)$ for some $0<\alpha<1$. Assume that $b(x)>0$, and that, having set $L=\Delta+a(x)$, we have

$$
\lambda_{1}^{L}(M)<0 \text {. }
$$

Then the equation

$$
\Delta u+a(x) u-b(x) u^{\sigma}=0, \quad \sigma>1,
$$

has a unique minimal $C^{2}$ positive solution.

As mentioned in the Introduction, if we assume that the function $a(x)$ is positive somewhere on $M$, then the condition $\lambda_{1}^{L}(M)<0$ amounts to the fact that $\mu=1$ is larger than the principal eigenvalue $\lambda_{*}$ of the problem $\Delta u+\lambda a(x) u=0$ on $M$, and Theorem 2.1 compares with the existence results in [AB], Theorem 2.2 and [DM2], Theorem 1 (for the latter, see also the remark after Theorem 2.3).

The proof of Theorem 2.1 uses the method of super- and sub-solutions, and the main task is the construction of a sub-solution, which is where the assumption on the sign of $\lambda_{1}^{L}(M)$ plays a crucial role. In the main result of this section, Theorem 2.10 below, we describe conditions not expressed in terms of the sign of $\lambda_{1}^{L}(M)$, for which one can guarantee existence of a solution.

Our first result, Theorem 2.4 below, states that, if one has a global sub-solution of (2.1) and the set where the non-negative coefficient $b$ is suitably small, it is always possible to prove the existence of a maximal solution.

The idea of the proof consists in applying the method of sub- and super-solutions to a sequence of boundary value problems on domains which exhaust the manifold.

Since a global sub-solution of (2.1) is given, one first needs to find a local supersolution. If $b(x)$ is strictly positive a sufficiently large constant will do. Even if this is not the case, a super-solution can be found provided the set where $b(x)$ vanishes is small (see Theorem 2.3 below).

To apply the approximation method it is also crucial that the approximating sequence is monotonic, and this follows from the next comparison result.

Proposition 2.2. Let $D \subset M$ be an open set with smooth boundary $\partial D$, and assume that $a(x), b(x)$ are functions in $C(D)$, and that $b(x)$ is non-negative. Let $u, v \in C^{0}(\bar{D}) \cap C^{2}(D)$ be solutions on $D$ of

$$
\Delta u+a(x) u-b(x) u^{\sigma} \geq 0
$$

and

$$
\Delta v+a(x) v-b(x) v^{\sigma} \leq 0,
$$

respectively, with $u \geq 0, v>0$. If $u \leq v$ on $\partial D$, then $u \leq v$ on $D$.

Proof. The proof is modelled on that of the generalized maximum principle. Set $w=u / v$. A computation shows that

$$
\Delta w \geq b(x)\left(u^{\sigma-1}-v^{\sigma-1}\right) w-2\langle\nabla w, \nabla \log v\rangle .
$$


Assume by contradiction that $u>v$ somewhere in $D$, so that, for sufficiently small $\epsilon>0$

$$
\Omega_{\epsilon}=\{x \in D: w(x)>1+\epsilon\} \neq \emptyset .
$$

Then $\Omega_{\epsilon} \Subset D$, and since $u>v$ on $\Omega_{\epsilon} \sigma \geq 1$ and $b$ is non-negative,

$$
\Delta w+2\langle\nabla w, \nabla \log v\rangle \geq 0 \text { on } \Omega_{\epsilon} .
$$

Since $w=1+\epsilon$ on $\partial \Omega_{\epsilon}$, by the maximum principle, $w \leq 1+\epsilon$ on $\Omega_{\epsilon}$, a contradiction.

We remark that Proposition 2.2 holds if the functions $u$ and $v$ are only assumed to be in $C^{1}(D) \cap C^{0}(\bar{D})$, provided we interpret (2.2) and (2.3) in the weak sense.

Before stating Theorem 2.4, we also need to make precise the sense in which the set where $b(x)$ vanishes is small.

Let $a(x) \in C^{0}(M)$ and let $L=\Delta+a(x)$. If $\Omega$ is a non-empty open set, the first Dirichlet eigenvalue $\lambda_{1}^{L}(\Omega)$ is variationally defined as in Section 1 by means of the formula

$$
\lambda_{1}^{L}(\Omega)=\inf \left\{\int_{\Omega}|\nabla \phi|^{2}-a(x) \phi^{2}: \phi \in H_{o}^{1}(\Omega), \int_{\Omega} \phi^{2}=1\right\},
$$

and, if $\Omega$ is bounded and both $\Omega$ and $a$ are sufficiently regular, the infimum is attained and there exists a unique normalized eigenfunction $v$ defined on $\Omega$ satisfying

$$
\left\{\begin{array}{l}
\Delta v+a(x) v+\lambda_{1}^{L}(\Omega) v=0 \quad \text { on } \Omega, \\
v>0 \text { on } \Omega, v \equiv 0 \text { on } \partial \Omega .
\end{array}\right.
$$

We extend the definition to an arbitrary bounded subset $S$ of $M$, by setting

$$
\lambda_{1}^{L}(S)=\sup \lambda_{1}^{L}(\Omega),
$$

where the supremum is taken over all open bounded sets with smooth boundary $\Omega$ such that $S \subset \Omega$. Note that, by definition, if $S=\emptyset$, then $\lambda_{1}^{L}(S)=+\infty$. Finally, if $S$ is an unbounded subset of $M$, we define

$$
\lambda_{1}^{L}(S)=\inf \lambda_{1}(D \cap S),
$$

where the infimum is taken over all bounded open sets with smooth boundary. Note that if $\left\{D_{n}\right\}$ is an increasing sequence of open sets with smooth boundary which exhausts $M$, then, by domain monotonicity, $\lambda_{1}^{L}(S)=\lim _{n} \lambda_{1}\left(D_{n} \cap S\right)$.

Since the first Dirichlet eigenvalue of the Laplacian of a ball $B_{r}$ grows like $r^{-2}$ as $r \rightarrow 0, \lambda_{1}^{L}\left(B_{r}\right)>0$ provided $r$ is sufficiently small, and one may think that the condition $\lambda_{1}^{L}(S)>0$ expresses the fact that $S$ is small in a spectral sense.

This notion of smallness is appropriate for our purposes. Indeed, P. Li, L.-F. Tam and D. Yang, LTY], have established the following relationship between the first eigenvalue of the set where $b(x)$ vanishes, and the existence of a non-trivial super-solution of equation (2.1).

Theorem 2.3. Let $a(x)$ and $b(x)$ be Hölder continuous functions on $M$, with $b(x) \geq$ 0 on $M$, and let $S_{o}=\{x \in M: b(x)=0\}$. Let $\Omega$ be a bounded open domain in $M$, and let $L=\Delta+a(x)$. If equation (2.1) has a positive super-solution on $\Omega$, then $\lambda_{1}^{L}\left(\Omega \cap S_{o}\right) \geq 0$.

Conversely, if $\lambda_{1}^{L}\left(\Omega \cap S_{o}\right)>0$, then (2.1) has a positive super-solution on $\Omega$. 
We remark that using Theorem 2.3 we may improve Theorem 2.1 above by replacing the assumption that $b(x)>0$ on $\mathrm{M}$ with the assumption that $b$ is nonnegative, and its zero set $S_{o}$ is such that $\lambda_{1}^{L}\left(S_{o}\right)>0$. Indeed, the strict positivity of $b$ is only used to guarantee that on every bounded domain a suitably large constant is a super-solution of equation (2.1) (see [BRS2, p.184).

Note that, if we assume that $S_{o}$ is a bounded domain with smooth boundary such that $a(x)$ is positive somewhere in $S_{o}$, then the condition $\lambda_{1}^{L}\left(S_{o}\right)>0$ amounts to the fact that 1 is strictly smaller than the principal eigenvalue of the problem $\Delta u+\lambda a(x) u=0$ with Dirichlet boundary conditions. Recalling the remark after the statement of Theorem 2.1 we conclude that if $\lambda_{*}<1<\lambda_{1}\left(L_{\mu}, S_{o}\right)$, then equation (2.1) has a unique positive minimal solution on $M$. This again compares with Theorem 1 in DM2].

We are now ready to state

Theorem 2.4. Let $a(x), b(x) \in C_{\text {loc }}^{0, \alpha}(M)$ for some $0<\alpha \leq 1$. Assume that $b(x)$ is non-negative, and strictly positive off a compact set, and that, denoting with $S_{0}$ the set where $b(x)$ vanishes, we have $\lambda_{1}^{L}\left(S_{o}\right)>0$. If $u_{-} \in C^{0}(M) \cap H_{l o c}^{1}(M), u_{-} \geq 0$, $u_{-} \not \equiv 0$, is a global sub-solution of equation (2.1), then (2.1) has a maximal positive $C^{2}$ solution.

Proof. Let $D_{k}$ be an increasing exhaustion of $M$ by an open domain with smooth boundary such that $S_{0} \subset D_{k} \subset \bar{D}_{k} \subset D_{k+1}$ for every $k$. Fix $k$ in $\mathbb{N}$. Since $\lambda_{1}^{L}\left(S_{o}\right)>0$, by Theorem 2.3 there exists a $C^{2}$ positive function $v$ satisfying

$$
\Delta v+a(x) v-b(x) v^{\sigma} \leq 0 \text { on } D_{k+1} .
$$

Since $\bar{D}_{k}$ is compactly contained in $D_{k+1}, \inf _{D_{k}} v>0$ and $u_{-}$is bounded on $\bar{D}_{k}$. Thus, given $n \geq \max _{\bar{D}_{k}} u_{-}$, there exists $C>0$ large enough that the function $v_{+}=C v$ satisfies

$$
\left\{\begin{array}{l}
\Delta v_{+}+a(x) v_{+}-b(x) v_{+}^{\sigma} \leq 0 \quad \text { on } D_{k}, \\
v_{+} \geq n \geq \max _{\bar{D}_{k}} u_{-} \text {on } \partial D_{k} \text { and } v_{+} \geq u_{-} \text {on } \bar{D}_{k} .
\end{array}\right.
$$

The monotone iteration scheme yields a solution $u_{k, n}$ of the boundary value problem

$$
\begin{cases}\Delta u+a(x) u-b(x) u^{\sigma}=0 & \text { on } B_{k}, \\ u=n & \text { on } \partial B_{k} .\end{cases}
$$

We now show that the sequence $\left\{u_{k, n}\right\}$ is uniformly bounded with respect to $n \in \mathbb{N}$ on compact subsets of $D_{k}$.

Assume first that $K$ is a compact subset of $D_{k}$ which does not intersect $S_{0}$. Then we may find a positive constant $b_{o}$ and a finite number of disjoint open balls $B_{i}$ which cover $K$ such that $b(x) \geq b_{o}$ on each $B_{i}$. Applying Lemma 2.6 in [PRS2] we deduce that there exists a constant $C_{1}=C_{1}(K)>0$ such that

$$
u_{k, n}(x) \leq C_{1} \quad \forall x \in K, \forall n .
$$

Next we show that $u_{k, n}$ is uniformly bounded in a neighborhood of $S_{0}$. By definition there exist open sets with smooth boundary $\Omega$ and $\Omega^{\prime}$ such that $S_{0} \subset$ $\Omega \subset \bar{\Omega} \subset \Omega^{\prime} \subset \bar{\Omega}^{\prime} \subset D_{k}$ and $\lambda_{1}^{L}\left(\Omega^{\prime}\right)>0$.

Note that since $\partial \Omega$ is a compact subset of $D_{k}$ which does not intersect $S_{0}$, there exists a constant $C_{2}$ such that $u_{k, n} \leq C_{2}$ on $\partial \Omega$. Next, let $\phi$ be a positive eigenfunction for $L$ belonging to $\lambda_{1}^{L}\left(\Omega^{\prime}\right)>0$. Since $\phi$ is positive on $\Omega^{\prime}$, it is bounded 
away from zero on $\bar{\Omega}$ and there exists a positive constant $c$ such that $c \phi>C_{2}$ on $\bar{\Omega}$.

Note that

$$
\Delta(c \phi)+a(x)(c \phi)=-\lambda_{1}^{L}\left(\Omega^{\prime \prime}\right)(c \phi)<0
$$

while

$$
\Delta u_{k, n}+a(x) u_{k, n}=b(x) u_{k, n}^{\sigma} \geq 0
$$

on $\Omega$, and $u_{k, n} \leq C_{2}<c \phi$ on $\partial \Omega$.

We claim that $u_{k, n} \leq c \phi$ on $\Omega$. Indeed, assume that this is not so, and let $A=\left\{x \in \Omega^{\prime}: u_{k, n}-c \phi>0\right\}$. Then $A$ is non-empty and $\bar{A} \subset \Omega$, and we deduce that $w=u_{k, n}-c \phi$ attains a positive maximum in $A$. On the other hand, $w$ satisfies

$$
\begin{cases}\Delta w+a(x) w \geq 0 & \text { in } A, \\ w=0 & \text { on } \partial A,\end{cases}
$$

and therefore, by the generalized maximum principle, $w / \phi$ is constant on $A$, and since it vanishes on $\partial A$ we conclude that $w / \phi=0$ on $A$, that is, $w=0$ on $A$, a contradiction.

Thus $u_{k, n} \leq c \phi \leq C_{2}$ on $\bar{\Omega}$ and it follows easily that $u_{k, n}$ is uniformly bounded on compact subset of $D_{k}$.

By interior elliptic estimates, a subsequence of $u_{k, n}$ converges in $C_{l o c}^{2}$ to a solution $u_{k}^{\infty}$ of

$$
\begin{cases}\Delta u+a(x) u-b(x) u^{\sigma}=0 & \text { on } D_{k}, \\ u=+\infty & \text { on } \partial D_{k} .\end{cases}
$$

We consider the sequence $\left\{u_{k}^{\infty}\right\}$. Clearly, $u_{k}^{\infty} \geq u_{-}>0$, and an exhaustion argument and Proposition 2.2 show that

$$
u_{k+1}^{\infty} \leq u_{k}^{\infty} \quad \text { on } \bar{D}_{k} .
$$

Since $\left\{u_{k}^{\infty}\right\}$ is monotone non-increasing, it converges to a function $u$ which solves (2.1) and satisfies $u \geq u_{-} \geq 0, u_{-} \not \equiv 0$ on $M$. If $u_{1}$ is another positive solution of (2.1) on $M$, then $u_{1} \leq u_{k}^{\infty}$ by Proposition 2.2 and therefore $u_{1} \leq u$, thus proving the maximality of $u$. Finally, $u$ is strictly positive, for otherwise the non-negative function $u_{-}$would attain a zero minimum, thus violating the minimum principle (GT, p. 35).

It is worth pointing out the following consequence of Proposition 2.2.

Proposition 2.5. Let $a(x), b(x) \in C^{0}(M), 0<\alpha<1$, and $b(x) \geq 0, b(x) \not \equiv 0$. Then the problem

$$
\left\{\begin{array}{l}
\text { (i) } \Delta u+a(x) u-b(x) u^{\sigma}=0, \quad \sigma>1, \\
\text { (ii) } \lim _{r(x) \rightarrow+\infty} u(x)=L>0
\end{array}\right.
$$

has at most one positive $C^{2}$ solution.

Proof. Let $u, v$ be positive $C^{2}$ solutions of (2.8). Choose any $\epsilon>0$ and observe that, since $b$ is non-negative, the function $w_{\epsilon}=(1+\epsilon) v$ is a super-solution of (2.8) (i) satisfying

$$
\lim _{r(x) \rightarrow+\infty} w_{\epsilon}(x)=(1+\epsilon) L
$$


Fix $R_{0}>0$ sufficiently large, so that for every $R>R_{0}$ we have $b(x) \not \equiv 0$ on $B_{R}$ and $w_{\epsilon}-u>0$ on $\partial B_{R}$. The latter is possible because of the limit relations (2.8) (ii) and (2.9). It follows from Proposition 2.2 that $w_{\epsilon} \geq u$ on $B_{R}$ for every $R \geq R_{0}$. Thus, $u(x) \leq(1+\epsilon) v(x)$ on $M$, and since $\epsilon>0$ was arbitrary, $u \leq v$ on $M$. Interchanging $u$ and $v$ yields the reverse inequality, and equality follows.

We remark that the assumption $L>0$ in the statement of the proposition cannot be weakened to $L \geq 0$. Indeed, in BRS2, pp. 214-215, it is shown that on $m$ dimensional hyperbolic space $\mathbb{H}^{m}$, equation (2.8) with $a(x) \equiv m(m-2) / 4, b(x) \equiv 1$ and $\sigma=(m+2) /(m-2)$ has a family of positive distinct radial solutions which tend to zero at infinity at the same rate.

It may also be worth noting that the assumptions on $u$ and $v$ in the uniqueness result obtained above may be weakened provided some conditions on the coefficients $a$ and $b$ and on the volume growth of the manifold are imposed.

Theorem 2.6. Let $a(x), b(x) \in C^{0}(M)$ and assume that, for some $C>0$ and $0 \leq \mu<2$,

$$
\text { (i) } b(x) \geq C(1+r(x))^{-\mu} \quad \text { and } \quad \text { (ii) } \sup _{M} \frac{a_{-}}{b}<+\infty \text {. }
$$

Assume that $u$ and $v$ are $C^{2}$ non-negative solutions of

$$
\Delta u+a(x) u-b(x) u^{\sigma} \geq 0 \geq \Delta v+a(x) v-b(x) v^{\sigma}, \quad \sigma>1,
$$

on $M$, satisfying

$$
\liminf _{r(x) \rightarrow \infty} v(x)>0, \quad \limsup _{r(x) \rightarrow \infty} u(x)<+\infty .
$$

If

$$
\frac{r^{1-\mu}}{\log \operatorname{vol} B_{r}} \notin L^{1}(+\infty)
$$

then $u \leq v$.

Proof. Note first of all that, by the maximum principle (see GT, p. 35), $v$ is strictly positive, and therefore, by the lim inf condition, it is bounded away from 0 on $M$. Also, $u$ is bounded above on $M$. We may assume that $u$ is not identically zero, or else there is nothing to prove. Thus, $\beta=\sup _{M} \frac{u}{v}$ is finite and strictly positive. The conclusion of the theorem amounts to saying that $\beta \leq 1$. Assume by contradiction that $\beta>1$, and let $\phi=u-\beta v$.

Clearly $\phi \leq 0$. We claim that $\sup _{M} \phi=0$. Indeed, there exists a sequence $x_{n}$ such that $\frac{u\left(x_{n}\right)}{v\left(x_{n}\right)} \rightarrow \beta>0$, and since $u\left(x_{n}\right)$ is bounded above, so must be $v\left(x_{n}\right)$ (for else $\beta=0)$, and then

$$
\phi\left(x_{n}\right)=v\left(x_{n}\right)\left(\frac{u\left(x_{n}\right)}{v\left(x_{n}\right)}-\beta\right) \rightarrow 0 \quad \text { as } n \rightarrow+\infty,
$$

as claimed.

We write

$$
\begin{aligned}
\Delta \phi & \geq \Delta(u-\beta v)=-a(x)[u-\beta v]+b(x)\left[u^{\sigma}-\beta v^{\sigma}\right] \\
& =-a(x) \phi+b(x)\left[u^{\sigma}-(\beta v)^{\sigma}\right]+b(x) v^{\sigma}\left[\beta^{\sigma}-\beta\right] .
\end{aligned}
$$

By the mean value theorem we have

$$
\left[u^{\sigma}-(\beta v)^{\sigma}\right](x)=h(x)(u-\beta v)=h(x) \phi,
$$


where

$$
h(x)=\frac{\sigma}{u(x)-\beta v(x)} \int_{\beta v(x)}^{u(x)} t^{\sigma-1} d t
$$

is continuous and non-negative on $M$. Further, since $u$ is bounded above, it follows that $h$ is bounded above by a constant $H$ on the set $\{x: \phi(x)>-1\}$. Also, since $\beta>1, \sigma>1$ and $v$ is bounded away from zero,

$$
v^{\sigma}\left[\beta^{\sigma}-\beta\right] \geq 2 c>0,
$$

for some positive constant $c$. Inserting the above expressions in (2.12), noting that, since $\phi$ is non-positive, $-a(x) \phi \geq a_{-}(x) \phi$, and dividing through by $b(x)$, we obtain

$$
\frac{1}{b} \Delta \phi \geq\left(\frac{a_{-}}{b}+h(x)\right) \phi+2 c .
$$

Now let $\epsilon \in(0,1)$ be such that

$$
\left(\sup \frac{a_{-}}{b}+H\right) \epsilon<c
$$

and let $\Omega_{\epsilon}=\{x: \phi(x)>-\epsilon\}$, which is not empty since $\sup \phi=0$. Then

$$
\frac{1}{b(x)} \Delta \phi \geq c>0 \quad \text { on } \Omega_{\epsilon} \text {. }
$$

On the other hand, since the volume growth condition (2.11) holds, and $b(x)$ satisfies the lower estimate (2.10) (i), Proposition 3.17 in PRS2 applies, and the weak maximum principle holds, namely,

$$
\inf _{\Omega_{\epsilon}} \frac{1}{b(x)} \Delta \phi \leq 0
$$

thus yielding the required contradiction.

As an immediate corollary we have

Corollary 2.7. Let $a$ and $b$ satisfy the conditions listed in Theorem 2.6, and let $u$ and $v$ be non-negative solutions of

$$
\Delta u+a(x) u-b(x) u^{\sigma}=0 .
$$

If both $u$ and $v$ satisfy the condition

$$
0<\liminf _{r(x) \rightarrow \infty} u(x) \leq \limsup _{r(x) \rightarrow \infty} u(x)<+\infty,
$$

and (2.11) holds, then $u=v$.

As observed above, condition (2.13) amounts to requiring that $u$ and $v$ are bounded and bounded away from zero on $M$. We also note that the family of functions mentioned in the remark that follows Proposition 2.5] also shows that uniqueness fails if we do not assume that the lim inf of $u$ and $v$ are strictly positive.

The following geometric consequence is worth mentioning.

Corollary 2.8. Let $(M,\langle\rangle$,$) be a complete Riemannian manifold of dimension$ $m \geq 3$ and scalar curvature $s(x)$ satisfying

$$
s(x) \leq-C(1+r(x))^{-\mu}
$$

for some constants $C>0$ and $0 \leq \mu<2$. Assume that (2.11) holds. Then any conformal diffeomorphism of $M$ into itself which preserves the scalar curvature and whose stretching factor $u$ satisfies (2.13) is an isometry. 
Proof. Let $\phi: M \rightarrow M$ be a conformal diffeomorphism. Then $\phi^{\star}\langle\rangle=,u^{\frac{4}{m-2}}\langle$,$\rangle ,$ where $u$ is the stretching factor. Since $\phi$ preserves the scalar curvature, $u$ is a solution of

$$
c_{m} \Delta u-s(x) u+s(x) u^{\frac{m+2}{m-2}}=0
$$

(see, e.g., [PRS1, p. 1319 ff.), and the result follows at once from Corollary 2.7

We remark that here we require that $u$ is bounded above and away from zero, so that the conformal diffeomorphism $\phi$ is a quasi-isometry. By contrast, in PRS1, Corollary $3.4, \phi$ is not assumed to be a quasi-isometry, but the scalar curvature $s(x)$ is assumed to be bounded below.

We now proceed with the main result of this section, Theorem 2.10, where we show that, under suitable assumptions on the coefficients, equation (2.1) has a globally defined positive sub-solution, and therefore, a maximal positive solution. The proof is based on the method of super and sub-solutions. This is achieved by constructing a sub-solution inside and outside a suitable ball in such a way that they can be glued together to yield a global sub-solution.

We begin with the following lemma, which will be the key ingredient in the construction of a sub-solution in the complement of a ball.

Lemma 2.9. Let $A(r), B(r) \in C^{0}([0,+\infty))$ with $A(r) \geq 0$ and $B(r)>0$ on $[0,+\infty)$. Let $g$ be a non-decreasing smooth function on $[R,+\infty)$, for some $R>0$. Then, given $T>0$ and $\sigma>1$, the problem

$$
\left\{\begin{array}{l}
\alpha^{\prime \prime}+(m-1) \frac{g^{\prime}}{g} \alpha^{\prime}-A(r) \alpha-B(r) \alpha^{\sigma}=0 \text { on }(R, R+T), \\
\alpha(R)=\alpha_{o}, \alpha(R+T)=0
\end{array}\right.
$$

has a $C^{2}$ solution $\alpha$ on $[R, R+T]$. Furthermore $\alpha>0$ and $\alpha^{\prime}<0$ in $[R, R+T)$ and for every $T_{o} \in(0, T]$ the following estimate holds:

$$
\left|\alpha^{\prime}(R)\right| \leq\left\{\frac{g\left(R+T_{o}\right)}{g(R)}\right\}^{m-1}\left\{T_{o} \max _{\left[R, R+T_{o}\right]}\left[A(s)+B(s) \alpha_{o}^{\sigma-1}\right]+\frac{1}{T_{o}}\right\} \alpha_{o} .
$$

Proof. We first show the existence of a solution $\alpha$ to (2.14). We extend $g$ to a smooth, non-decreasing function on $[0,+\infty)$ satisfying $g^{\prime}(0)=1$ and $g^{(2 k)}(0)=0$ for every $k \in \mathbb{N}$ and consider the model manifold $M=\mathbb{R}^{m}$ with the metric given in polar coordinates by

$$
\langle,\rangle=d r^{2}+g(r)^{2} d \theta^{2}
$$

Note that by the conditions imposed on the function $g$, the metric originally defined on $\mathbb{R}^{m} \backslash\{0\}$ extends to a smooth metric on the whole of $\mathbb{R}^{m}$.

Let $R_{1}$ and $\bar{R}$ be such that $0<R_{1}<R<R+T<\bar{R}$, and let $\psi$ be a smooth radial cutoff function such that $0 \leq \psi \leq 1, \psi \equiv 1$ on $B_{R_{1}}$, and $\psi \equiv 0$ on $M \backslash \bar{B}_{R+T}$. Define

$$
\bar{a}(x)=-\psi(x) A(r(x))+N(1-\psi(x)),
$$

where $N$ is constant, and let $\bar{L}$ be the Schrödinger operator $L=\Delta+\bar{a}(x)$. We claim that if $N$ is sufficiently large, then

$$
\lambda_{1}^{\bar{L}}\left(B_{\bar{R}}\right)<0 .
$$

Indeed, let $u$ be any smooth function satisfying $u>0$ in $B_{\bar{R}}$ and $u=0$ on $\partial B_{\bar{R}}$. Then

$$
\int_{B_{\bar{R}}}|\nabla u|^{2}-\bar{a}(x) u^{2}=\int_{B_{\bar{R}}}\left[|\nabla u|^{2}+\psi(x) A(r(x)) u^{2}\right]-N \int_{B_{\bar{R}}}(1-\psi) u^{2},
$$


and since $(1-\psi) u^{2}>0$ in $B_{\bar{R}} \backslash B_{R+T}$, the right hand side may be made negative provided $N$ is large enough. The claim now follows from the variational characterization of $\lambda_{1}^{\bar{L}}\left(B_{\bar{R}}\right)$.

Let $\phi$ be the radial, normalized eigenfunction belonging to $\lambda_{1}^{\bar{L}}\left(B_{\bar{R}}\right)$. By definition

$$
\int_{B_{\bar{R}}}|\nabla \phi|^{2}-\bar{a}(x) \phi^{2}=\lambda_{1}^{\bar{L}}\left(B_{\bar{R}}\right)<0,
$$

so that, if $\gamma>0$ is sufficiently small,

$$
\int_{B_{\bar{R}}}|\nabla \phi|^{2}-\bar{a}(x) \phi^{2}+\gamma B(r(x)) \phi^{2}=\lambda_{1}^{\bar{L}}\left(B_{\bar{R}}\right)+\gamma \int_{B_{\bar{R}}} B(r(x)) \phi^{2}<0 .
$$

Thus, if we denote by $\tilde{L}=L+\bar{a}(x)-\gamma B(r(x))$, then

$$
\lambda_{1}^{\tilde{L}}\left(B_{\bar{R}}\right)<0 .
$$

If $\xi$ is a positive radial eigenfunction belonging to $\lambda_{1}^{\tilde{L}}\left(B_{\bar{R}}\right)$, so that

$$
\Delta \xi+\bar{a}(x) \xi=\gamma B(r(x)) \xi-\lambda_{1}^{\tilde{L}}\left(B_{\bar{R}}\right) \xi \geq \gamma B(r(x)) \xi \text { on } B_{\bar{R}},
$$

then the function $v_{-}=\mu \xi$ satisfies

$$
\left\{\begin{array}{l}
\Delta v_{-}+\bar{a}(x) v_{-}-B(r(x)) v_{-}^{\sigma} \geq B(r(x)) \mu \xi\left[\gamma-(\mu \xi)^{\sigma-1}\right] \geq 0 \text { in } B_{\bar{R}}, \\
v_{-}>0 \text { on } B_{\bar{R}}, \quad v_{-} \equiv 0 \text { on } \partial B_{\bar{R}}
\end{array}\right.
$$

provided $0<\mu<\gamma^{1 /(\sigma-1)}\left(\sup _{B_{\bar{R}}} \xi\right)^{-1}$.

On the other hand, since $B(r)>0$, a sufficiently large positive constant $v_{+}$is a super-solution of the above problem, that is,

$$
\left\{\begin{array}{l}
\Delta v_{+} \bar{a}(x) v_{+}-B(r(x)) v_{+}^{\sigma} \leq 0 \text { in } B_{\bar{R}} \\
v_{+}>0 \text { on } \partial B_{\bar{R}}
\end{array}\right.
$$

and by the monotone iteration scheme there exists a solution $w$ to the problem

$$
\left\{\begin{array}{l}
\Delta w+\bar{a}(x) w-B(r(x)) w^{\sigma}=0, w>0 \text { in } B_{\bar{R}} \\
w \equiv 0 \text { on } \partial B_{\bar{R}}
\end{array}\right.
$$

Note that $w$ is radial, since the monotone iteration scheme produces radial solutions in a radial setting. Moreover, since $\bar{a}(x)>-A(r(x))$ and $\inf _{\bar{B}_{R+T}} w>0$, if $c$ is sufficiently large, then the function $w_{+}=c w$ satisfies

$$
\left\{\begin{array}{l}
\Delta w_{+}-A(r(x)) w_{+}-B(r(x)) w_{+}^{\sigma} \leq 0 \text { in } B_{\bar{R}}, \\
w_{+} \geq 0 \text { on } \partial B_{R+T} \cup \partial B_{R}
\end{array}\right.
$$

and since $w_{-} \equiv 0$ is a sub-solution of the problem, applying once again the monotone iteration scheme produces a radial non-negative $C^{2}$ solution $u$ of

$$
\left\{\begin{array}{l}
\Delta u+-A(r(x)) u-B(r(x)) u^{\sigma}=0 \text { in } B_{R+T} \backslash \bar{B}_{R}, \\
u \geq 0, u=\alpha_{o} \text { on } \partial B_{R}, u=0 \text { on } \partial B_{R+T}
\end{array}\right.
$$

Since $u$ satisfies

$$
\Delta u-\left(A(r(x))+B(r(x)) u^{\sigma-1}\right) u=0,
$$

by the strong maximum principle (see [GT], p. 35) we deduce that $u>0$ in $B_{R+T} \backslash \bar{B}_{R}$. Since $u$ is radial, we deduce that $u(x)=\alpha(r(x))$ with $\alpha$ satisfying (2.14). 
We now show that $\alpha^{\prime}<0$ in $[R, R+T)$. Indeed, assume this is not so. Since $\alpha(r)>0=\alpha(R+T)$, then there exists $r_{o} \in[R, R+T)$ such that $\alpha^{\prime}\left(r_{o}\right)=0$. Integrating (2.14) between $r_{o}$ and $r$ gives

$$
g(r)^{m-1} \alpha^{\prime}(r)=\int_{r_{o}}^{r} g(s)^{m-1} \alpha(s)\left[A(s)+B(s) \alpha(s)^{\sigma-1}\right] d s,
$$

and the integral on the right hand side is striclty positive for every $r>r_{o}$. Thus $\alpha^{\prime}(r)>0$ in $\left[r_{o}, R+T\right]$, contradicting $\alpha\left(r_{o}\right)>0, \alpha(R+T)=0$.

It remains to show that estimate (2.15) holds. Integrating (2.14) between $R+t_{1}$ and $R+t_{2}$ with $0 \leq t_{1}<t_{2} \leq T$ yields

$$
\begin{aligned}
g\left(R+t_{2}\right)^{m-1} \alpha^{\prime}\left(R+t_{2}\right)- & g\left(R+t_{1}\right)^{m-1} \alpha^{\prime}\left(R+t_{1}\right) \\
& =\int_{R+t_{1}}^{R+t_{2}} g(s)^{m-1} \alpha(s)\left[A(s)+B(s) \alpha(s)^{\sigma-1}\right] d s,
\end{aligned}
$$

and since the integrand is positive, we deduce that

$$
g\left(R+t_{1}\right)^{m-1} \alpha^{\prime}\left(R+t_{1}\right)<g\left(R+t_{2}\right)^{m-1} \alpha^{\prime}\left(R+t_{2}\right),
$$

whence, recalling that $\alpha^{\prime}<0$ and $g$ is non-decreasing,

$$
\frac{\alpha^{\prime}\left(R+t_{1}\right)}{\alpha^{\prime}\left(R+t_{2}\right)}>\left(\frac{g\left(R+t_{2}\right)}{g\left(R+t_{2}\right)}\right)^{m-1} \geq 1
$$

and therefore

$$
\alpha^{\prime}\left(R+t_{1}\right)<\alpha^{\prime}\left(R+t_{2}\right) \leq 0, \quad \forall 0 \leq t_{1}<t_{2} \leq T .
$$

Moreover, for every $T_{o} \in(0, T]$ there exists $t \in\left(0, T_{o}\right)$ such that

$$
\frac{\alpha_{o}}{T_{o}}=\frac{\alpha(R)-\alpha(R+T)}{T_{o}}=-\alpha^{\prime}(R+t)>-\alpha\left(R+T_{o}\right) .
$$

Writing (2.16) with $t_{1}=0, t_{2}=T_{o}$, and using the above inequality we obtain

$-g(R)^{m-1} \alpha^{\prime}(R) \leq g\left(R+T_{o}\right)^{m-1} \frac{\alpha_{o}}{T_{o}}+T_{o} g\left(R+T_{o}\right)^{m-1} \alpha_{o} \max _{\left[R, R+T_{o}\right]}\left[A(s)+B(s) \alpha_{o}^{\sigma-1}\right]$,

whence, for every $t \in[R, R+T]$,

$$
\left|\alpha^{\prime}(t)\right| \leq\left|\alpha^{\prime}(R)\right| \leq\left(\frac{g\left(R+T_{o}\right)}{g(R)}\right)^{m-1}\left\{\frac{1}{T_{o}}+T_{o} \max _{\left[R, R+T_{o}\right]}\left[A(s)+B(s) \alpha_{o}^{\sigma-1}\right]\right\} \alpha_{o},
$$

as required.

We are now ready to prove the main result of this section.

Theorem 2.10. Let $(M,\langle\rangle$,$) be a complete manifold, and assume that the differ-$ ential inequality

$$
\Delta r(x) \leq(m-1) \frac{g^{\prime}(r(x))}{g(r(x))}
$$

holds pointwise in the complement of the cut locus Cut of o, for some function $g \in C^{\infty}([0,+\infty))$, with $g^{(2 k)}(0)=0$ if $k=0,1,2, \ldots, g^{\prime}(0)=1, g(t)>0$ and $g^{\prime}(t) \geq 0$ if $t>0$. Let $a(x), b(x) \in C_{\text {loc }}^{0, \alpha}(M)(0<\alpha \leq 1)$, and assume that $b(x)$ 
satisfies the conditions in Theorem 2.4. Suppose that we can choose $R>0$ and $T_{o}>0$ in such a way that

$$
R \inf _{B_{R}} a>\left(1+\sup _{B_{R}} r \Delta r\right)\left(\frac{1}{T_{o}}+T_{o} \max _{\bar{B}_{R+T_{o}} \backslash B_{R}} a_{-}\right)\left(\frac{g\left(R+T_{o}\right)}{g(R)}\right)^{m-1} .
$$

Then the equation

$$
\Delta u+a(x) u-b(x) u^{\sigma}=0, \quad \sigma>1,
$$

has a maximal positive solution on $M$.

Remark. Observe that, by the Laplacian Comparison Theorem, see, e.g. GW], or BRS2, Appendix, the validity of an inequality of the form (2.17) can be deduced from appropriate lower bounds for the radial Ricci curvature. To compare with the existence theorems in the Euclidean setting that can be found in the literature, we note that, on $\mathbb{R}^{m}$, (2.17) holds with $g(r)=r$, while if we assume an "almost Euclidean behavior", namely, that the radial Ricci curvature satisfies an estimate

$$
\text { Ricc } \geq-(m-1) B\left(1+r^{2}\right)^{-1},
$$

then (2.17) holds with $g(r)=r^{B^{\prime}}$, where $B^{\prime}=\left[1+\sqrt{1+4 B^{2}}\right] / 2$. We note in passing that the assumption on the Ricci curvature does not imply that the manifold is quasi-isometric to Euclidean space. If we assume instead that

$$
\text { Ricc } \geq-(m-1) B
$$

so that, loosely speaking, the reference model is hyperbolic space of constant negative sectional curvature, then (2.17) holds with the choice $g(r)=\frac{1}{\sqrt{B}} \sinh (\sqrt{B} r)$.

We also note that condition (2.18) is satisfied if $a(x)$ is sufficiently large near the origin $o$ and non-negative in a suitably large ball. Further, up to choosing a different reference point, it suffices to assume that $a(x)$ has a positive spike somewhere. Of course (2.17) should then be written with respect to the new origin centered at the spike. Observe however that if we assume, e.g., that the Ricci curvature is bounded from below by a negative constant, then the validity of (2.17) is independent of the chosen origin.

Proof. According to Theorem 2.4 it suffices to show that (2.19) has a non-negative, non-identically zero global sub-solution. This will be obtained by joining suitable radial local sub-solutions. We set

$$
\min _{\partial B_{r}} a(x)=A(r)=A_{+}(r)-A_{-}(r), \quad B(r)=\max _{\partial B_{r}} b(x), \quad \text { and } \quad \tau=\max _{B_{r}} r \Delta r .
$$

Applying the previous lemma with $A_{-}(r)$ and $B(r)+\epsilon$ instead of $A(r)$ and $B(r)$, respectively, we deduce that for every $\alpha_{o}$ and $\epsilon>0$ there exists a solution $\alpha \in C^{2}\left(\left[R, R+T_{o}\right]\right)$ of the differential inequality

$$
\alpha^{\prime \prime}+(m-1) \frac{g^{\prime}}{g} \alpha^{\prime}-A_{-}(r) \alpha-B(r) \alpha^{\sigma} \geq 0,
$$

satisfying $\alpha(R)=\alpha_{o}, \alpha\left(R+T_{o}\right)=0, \alpha^{\prime}(r)<0$ on $\left[R, R+T_{o}\right)$ and

$$
0>\alpha^{\prime}(R)>-\left(\frac{g\left(R+T_{o}\right)}{g(R)}\right)^{m-1}\left\{\frac{1}{T_{o}}+T_{o} \max _{\left[R, R+T_{o}\right]}\left[A(s)+(B(s)+\epsilon) \alpha_{o}^{\sigma-1}\right]\right\} \alpha_{o} .
$$

Using the expression of the Laplacian of a radial function, and the inequalities (2.17), $a(x) \geq-A_{-}(r(x))$ and $b(x) \leq B(r(x))$ and $\alpha^{\prime} \leq 0$, it follows that the 
function $v(x)=\alpha(r(x))$ is Lipschitz in $B_{R+T_{o}} \backslash B_{R}$ and $C^{2}$ in the complement of the cut locus of $o$ where it satisfies the pointwise inequality

$$
\Delta v+a(x) v-b(x) v^{\sigma}=\alpha^{\prime \prime}+\alpha^{\prime} \Delta r+a(x) \alpha-b(x) \alpha^{\sigma} \geq 0 .
$$

To construct a sub-solution in the ball $B_{R}$ we consider the problem

$$
\left\{\begin{array}{l}
\beta^{\prime \prime}+\frac{\tau}{r} \beta^{\prime}+A(r) \beta-B(r) \beta^{\sigma} \geq 0 \quad \text { on }[0, R], \\
\beta>0, \beta^{\prime} \leq 0, \beta(R)=\alpha_{o}, \beta^{\prime}(R) \leq \alpha^{\prime}(R),
\end{array}\right.
$$

and we look for a solution of the form

$$
\beta(r)=\alpha_{o}\left(1+\left(R^{2}-r^{2}\right) \eta\right) \geq \alpha_{o}
$$

Note that $\beta^{\prime}(r)=-2 \eta R \alpha_{o}<0$, so, using (2.21), the condition $\beta^{\prime}(R) \leq \alpha^{\prime}(R)$ follows from

$$
2 \eta \geq \frac{1}{R}\left(\frac{g\left(R+T_{o}\right)}{g(R)}\right)^{m-1}\left\{\frac{1}{T_{o}}+T_{o} \max _{\left[R, R+T_{o}\right]}\left[A(s)+(B(s)+\epsilon) \alpha_{o}^{\sigma-1}\right]\right\} .
$$

On the other hand, a direct computation that uses $A(r) \geq \min _{B_{R}} a(x)$ and $B(r) \leq$ $\max _{B_{R}} b(x)$ shows that

$$
\beta^{\prime \prime}+\frac{\tau}{r} \beta^{\prime}+A(r) \beta-B(r) \beta^{\sigma} \geq \alpha_{o}\left\{\min _{B_{R}} a(x)-2 \eta(1+\tau)-\alpha_{o}^{\sigma-1} \eta^{\sigma} R^{\sigma} \max _{B_{r}} b(x)\right\}
$$

and the right hand side is non-negative provided

$$
2 \eta \leq \frac{1}{1+\tau}\left\{\min _{B_{R}} a-\alpha_{o}^{\sigma-1} \eta^{\sigma} R^{\sigma} \max _{B_{r}} b(x)\right\} .
$$

Using (2.18) we may choose $\alpha_{o}$ small enough that, for every $\eta \leq\left(\min _{B_{R}} a\right) /[2(1+\tau)]$ (which is the largest possible value of $\eta$ allowed by (2.26)) the right hand side of $(2.26)$ is greater than or equal to the right hand side of (2.25), and therefore choose $\eta$ in such a way that both (2.25) and (2.26) are satisfied. For such values of $\alpha_{o}$ and $\eta$, the function $\beta$ satisfies all the requirements. Proceeding as above one verifies that the function $w(x)=\beta(\alpha(r(x)))$ is Lipschitz in $B_{R}$ and $C^{2}$ in the complement of the cut locus where it satisfies the pointwise inequality

$$
\Delta w+a(x) w-b(x) w^{\sigma} \geq 0
$$

Now we define

$$
u_{-}(x)= \begin{cases}w & \text { on } B_{R}, \\ v & \text { on } B_{R+T_{o}} \backslash B_{R}, \\ 0 & \text { on } M \backslash B_{R+T_{o}},\end{cases}
$$

and claim that $u_{-} \in C^{0}(M) \cap H_{l o c}^{1}(M)$ is a weak global sub-solution of (2.19).

This is easily seen if we assume that $o$ is a pole of $M$, for then, given a positive test function $\varphi \in C_{c}^{\infty}(M)$, applying Green's second identity, and using the fact that $w$ and $v$ are pointwise sub-solutions of (2.19) in $B_{R}$ and $B_{R+T_{o}} \backslash \bar{B}_{R}$, respectively, 
we obtain

$$
\begin{aligned}
& \int_{M} u_{-} \Delta \varphi=\int_{M}\left\langle\nabla u_{-}, \nabla \varphi\right\rangle=\int_{B_{R}} \varphi \Delta w+\int_{B_{R+T_{o}} \backslash B_{R}} \varphi \Delta v \\
& +\int_{\partial B_{R}}(w-v)\langle\nabla \varphi, \nabla r\rangle-\varphi\langle\nabla(w-v), \nabla r\rangle+\int_{\partial B_{R+T_{o}}} v\langle\nabla \varphi, \nabla r\rangle-\varphi\langle\nabla v, \nabla r\rangle \\
\geq & \int_{M}\left(-a(x) u_{-}+b(x) u_{-}^{\sigma}\right) \varphi-\left(\beta^{\prime}(R)-\alpha^{\prime}(R)\right) \int_{\partial B_{R}} \varphi-\alpha^{\prime}\left(R+T_{o}\right) \int_{\partial B_{R+T_{o}}} \varphi
\end{aligned}
$$

and the claim follows from the inequalities $\beta^{\prime}(R) \leq \alpha^{\prime}(R), \alpha^{\prime}\left(R+T_{o}\right) \leq 0$.

In the case where the cut locus of $o$ is not empty, one can adapt an argument in PRS2, Lemma 2.2, as follows: we consider an exhaustion $\Omega_{n}$ of $M \backslash C u t_{o}$ by domains with smooth boundary, which are star-shaped with respect to $o$, so that, denoting by $\nu$ the outward unit normal, we have $\langle\nabla r, \nu\rangle>0$ on $\partial \Omega_{n}$. Since the part of $\partial B_{R}$ contained in $\Omega_{n}$ is smooth, we may also deform $\Omega_{n}$, if necessary, in such a way that, for every $n, \partial \Omega_{n}$ is transversal to $\partial B_{R}$ and $\partial B_{R+T_{o}}$.

Since $\nabla u_{-}$is locally bounded we have

$$
\int_{M} u_{-} \Delta \varphi=-\int_{M}\left\langle\nabla u_{-}, \nabla \varphi\right\rangle=-\lim _{n \rightarrow \infty} \int_{\Omega_{n}}\left\langle\nabla u_{-}, \nabla \varphi\right\rangle .
$$

We write $\Omega_{n}=\left(\Omega_{n} \cap B_{R}\right) \cup\left(\Omega_{n} \cap\left(B_{R+T_{o}} \backslash B_{R}\right)\right) \cup\left(\Omega_{n} \cap B_{R+T_{o}}^{c}\right)$, apply the divergence theorem, and use the fact that $v$ and $w$ are pointwise sub-solutions of (2.19) in the complement of the cut locus to obtain

$$
\begin{aligned}
-\int_{\Omega_{n}}\left\langle\nabla u_{-}, \nabla \varphi\right\rangle \geq & \int_{\Omega_{n}} \varphi\left[-a(x) u_{-}+b(x) u_{-}^{\sigma}\right] \\
& -\left(\int_{\partial\left(\Omega_{n} \cap B_{R}\right)} \varphi\langle\nabla w, \nu\rangle+\int_{\partial\left(\Omega_{n} \cap\left(B_{\left.\left.R+T_{o} \backslash B_{R}\right)\right)} \varphi\langle\nabla v, \nu\rangle\right) .\right.}\right.
\end{aligned}
$$

To conclude, note that, by the transversality assumption, up to sets of lower dimension, we have $\partial\left(\Omega_{n} \cap B_{R}\right)=\left(\Omega_{n} \cap \partial B_{R}\right) \cup\left(\partial \Omega_{n} \cap B_{R}\right)$ and similarly when $B_{R}$ is replaced by $B_{R+T_{o}} \backslash B_{R}$, so that the boundary integrals become

$$
\begin{aligned}
\left(\beta^{\prime}(R)-\alpha^{\prime}(R)\right) \int_{\Omega_{n} \cap \partial B_{R}} & \varphi+\alpha^{\prime}(R) \int_{\Omega_{n} \cap \partial B_{R+T_{o}}} \varphi \\
& +\int_{\partial \Omega_{n} \cap B_{R}} \varphi \beta^{\prime}\langle\nabla r, \nu\rangle+\int_{\partial \Omega_{n} \cap\left(B_{\left.R+T_{o} \backslash B_{R}\right)}\right.} \varphi \alpha^{\prime}\langle\nabla r, \nu\rangle
\end{aligned}
$$

and the first integral is non-positive because $\beta^{\prime}(R) \leq \alpha^{\prime}(R)$, while the last two are non-positive because $\alpha^{\prime}, \beta^{\prime} \leq 0$ and $\langle\nabla r, \nu\rangle \geq 0$ on $\partial \Omega_{n}$.

Remark 2.11. The above existence result is also relevant to the Yamabe problem, that is, the possibility of conformally deforming the assigned metric, with scalar curvature $s(x)$, to a new one with prescribed scalar curvature $K(x)$. Indeed assume that $m \geq 3$, and denote by $u^{2 /(m-2)}$ the conformal factor, so that the deformed metric is given by $\widetilde{\langle,\rangle}=u^{\frac{4}{(m-2)}}\langle$,$\rangle . Then the scalar curvature of \widetilde{\langle,\rangle}$ is $K(x)$ provided $u$ is a, necessarily positive, solution of the Yamabe equation

$$
c_{m} \Delta u-s(x) u+K(x) u^{\frac{m+2}{m-2}}=0,
$$

where $c_{m}=\frac{4(m-1)}{m-2}$, which is (2.19) with $a(x)=-c_{m}^{-1} s(x)$ and $b(x)=-c_{m}^{-1} K(x)$. 
By way of example, assume that Ricc $\geq-(m-1) B$, so that $s(x) \geq-m(m-1) B$, and, as noted above, (2.17) holds with $g(r)=(\sqrt{B})^{-1} \sinh (\sqrt{B} r)$. If we suppose that $s(x) \leq 0$ in a ball of radius $R+1$, then (2.18) holds, with $T_{o}=1$, provided

$$
R \sup _{B_{R}} s(x) \leq-c_{m}(1+(m-1) R \sqrt{B} \operatorname{coth}(\sqrt{B} R))\left(\frac{\sinh (\sqrt{B}(R+1))}{\sinh (\sqrt{B} R)}\right)^{m-1} .
$$

It is easy to see that if $\sqrt{B}>4(m-1) /[m(m-2)]$, then the above condition is verified provided $s(x)$ is sufficiently near $-m(m-1) B$ in the ball $B_{R}$ and $R$ is large enough. We note that there are situations (for instance when the sectional curvature is suitably pinched) where Theorem 2.10 is applicable, while Theorem 2.1 is not.

\section{NON-EXISTENCE RESUlts}

The purpose of this section is to prove a non-existence result for non-negative Lip $_{l o c}$ solutions of the differential inequality

$$
\Delta u+a(x) u-b(x) u^{\sigma} \geq 0 \quad \text { on } M .
$$

We begin with the following general

Theorem 3.1. Let $a(x), b(x) \in C^{0}(M)$ and assume that $b(x) \geq 0$. Let $H>0$, $K>-1$ and $A \in \mathbb{R}$ be constants satisfying

$$
\max \{-1, A\} \leq H(K+1)-1
$$

and suppose that there exists a positive Liploc solution of the differential inequality

$$
\Delta \varphi+H a(x) \varphi \leq-K \frac{|\nabla \varphi|^{2}}{\varphi} \quad \text { on } M .
$$

Then the differential inequality

$$
u \Delta u+a(x) u^{2}-b(x) u^{\sigma+1} \geq-A|\nabla u|^{2}, \quad \sigma \geq 1,
$$

has no non-negative Liploc solutions on $M$ satisfying

$$
\operatorname{supp} u \cap\{x \in M: b(x)>0\} \neq \emptyset
$$

and

$$
\left(\int_{\partial B_{r}} \varphi^{\frac{\beta+1}{H}(2-p)} u^{p(\beta+1)}\right)^{-1} \notin L^{1}(+\infty),
$$

for some $p>1$ and $\beta$ satisfying $\beta>-1, A \leq \beta \leq H(K+1)-1$.

Remark. We note that if $p=2$, then the non-integrability assumption (3.6) involves $u$ alone and reduces to

$$
\left(\int_{\partial B_{r}} u^{2(\beta+1)}\right)^{-1} \notin L^{1}(+\infty) .
$$

If $p \neq 2$, assumption (3.7) implies (3.6) if a suitable bound on $\varphi$ is available, e.g., if $p<2$ and $\varphi$ is bounded from above. A similar simplification occurs if $\varphi$ is bounded above, respectively below, by a radial function; see, e.g., Theorem 3.9 below.

We also remark that the inequality

$$
r-R=\int_{R}^{r} f^{1 / 2} f^{-1 / 2} \leq\left(\int_{R}^{r} f\right)^{1 / 2}\left(\int_{R}^{r} f^{-1}\right)^{1 / 2},
$$


valid for $f>0$, together with integration in polar coordinates, shows that condition (3.6) is implied by $\varphi^{\frac{2-p}{H}} u^{p} \in L^{\beta+1}(M)$.

Proof. The proof is a generalization and an improvement of that of Theorem 1.4 in PRS3.

Let $u \geq 0$ be a solution of (3.4) on $M$ satisfying (3.5) and (3.6). We set for ease of notation $\alpha=(\beta+1) / H$ and

$$
v=\varphi^{-\alpha} u^{\beta+1},
$$

and we claim that $v$ satisfies the differential inequality

$$
v \operatorname{div}\left(\varphi^{2 \alpha} v\right) \geq 0
$$

weakly on $M$. Since $\varphi^{2 \alpha} v^{p}=\varphi^{\frac{\beta+1}{H}(2-p)} u^{p(\beta+1)}, v$ satisfies the non-integrability condition in the statement of Theorem 1.1 in [PRS3, and it is therefore constant. Thus there exists a constant $C \geq 0$ such that

$$
u^{H}=C \varphi \text {. }
$$

Since $u$ is not identically zero by (3.5), $C>0$ and $u$ is strictly positive on $M$. We insert the expression of $\varphi$ in terms of $u$ in (3.3), divide by $C H u^{H-2}$ and subtract the result from (3.4) to obtain

$$
[A-H(K+1)+1]|\nabla u|^{2} \geq b(x) u^{\sigma+1} .
$$

Since the coefficient of $|\nabla u|^{2}$ is non-positive, by (3.2), we conclude that

$$
b(x) u^{\sigma+1} \leq 0,
$$

which contradicts (3.5).

To prove the claim, note first that the restrictions imposed on $\beta$, and Lemma 3.1 in PRS4 imply that $v \in C^{0}(M) \cap W_{l o c}^{1,2}(M)$. The claim amounts to showing that for every non-negative, compactly supported function $\rho \in W^{1,2}$ on $M$, we have

$$
I=\int\left[\left\langle\varphi^{2 \alpha} v \nabla v, \nabla \rho\right\rangle+\varphi^{2 \alpha}|\nabla v|^{2} \rho\right] \leq 0 .
$$

According to Lemma 3.2 in PRS4, the usual chain rule may be applied to compute the gradient of $u^{\beta+1}$, so that

$$
\nabla v=-\alpha \varphi^{-\alpha-1} u^{\beta+1} \nabla \varphi+(\beta+1) \varphi^{-\alpha} u^{\beta} \nabla u
$$

and

$$
\begin{aligned}
& I=(\beta+1) \int\left\langle\nabla u, u^{2 \beta+1} \nabla \rho\right\rangle-\alpha \int \varphi^{-1} u^{2 \beta+2}\langle\nabla \varphi, \nabla \rho\rangle \\
& +\int\left[(\beta+1)^{2} u^{2 \beta}|\nabla u|^{2}+\alpha^{2} u^{2 \beta+2} \frac{|\nabla \varphi|^{2}}{\varphi^{2}}\right] \rho-2 \alpha(\beta+1) \int u^{2 \beta+1}\left\langle\frac{\nabla \varphi}{\varphi}, \nabla u\right\rangle \rho .
\end{aligned}
$$

We first consider the first integral on the right hand side, and assume that $\beta<0$, the other case being easier. Since

$$
\left|\left(u^{2}+\epsilon\right)^{\beta} u \nabla u\right| \leq u^{2 \beta+1}|\nabla u|=u^{1+\beta} u^{\beta}|\nabla u| \in L_{l o c}^{1}
$$


by Lemma 3.1 in [PRS4, by the dominated convergence theorem,

$$
\begin{aligned}
& \int u^{2 \beta} u\langle\nabla u, \nabla \rho\rangle=\lim _{\epsilon \rightarrow 0+} \int\left(u^{2}+\epsilon\right)^{\beta} u\langle\nabla u, \nabla \rho\rangle \\
& =\lim _{\epsilon \rightarrow 0+}\left\{\int\left\langle\nabla u, \nabla\left[u\left(u^{2}+\epsilon\right)^{\beta} \rho\right]\right\rangle-\left(u^{2}+\epsilon\right)^{\beta} \frac{(2 \beta+1) u^{2}+\epsilon}{u^{2}+\epsilon}|\nabla u|^{2} \rho\right\} .
\end{aligned}
$$

According to (3.4), for every non-negative, compactly supported function $\lambda \in$ $W^{1,2}(M)$,

$$
\int\langle\nabla u, \nabla(\lambda u)\rangle \leq \int\left(a(x) u^{2}-b(x) u^{\sigma+1}+A|\nabla u|^{2}\right) \lambda .
$$

Applying the above inequality with $\lambda=\rho\left(u^{2}+\epsilon\right)^{\beta}$, and applying the dominated convergence theorem, we deduce that

$$
\lim _{\epsilon \rightarrow 0+} \int\left(u^{2}+\epsilon\right)^{\beta} \frac{(2 \beta+1) u^{2}+\epsilon}{u^{2}+\epsilon}|\nabla u|^{2} \rho=(2 \beta+1) \int u^{2 \beta}|\nabla u|^{2} \rho
$$

and

$$
\begin{aligned}
\lim _{\epsilon \rightarrow 0+} \int\left\langle\nabla u, \nabla\left[u\left(u^{2}+\epsilon\right)^{\beta} \rho\right]\right\rangle \leq & \lim _{\epsilon \rightarrow 0+} \int\left[a(x) u^{2}-b(x) u^{\sigma+1}+A|\nabla u|^{2}\right]\left(u^{2}+\epsilon\right)^{\beta} \rho \\
& =\int\left[a(x) u^{2 \beta+2}-b(x) u^{2 \beta \sigma+1}+A u^{2 \beta}|\nabla u|^{2}\right] \rho .
\end{aligned}
$$

Inserting into (3.10) we conclude that

$$
\int u^{2 \beta+1}\langle\nabla u, \nabla \rho\rangle \leq \int\left[a(x) u^{2 \beta+2}-b(x) u^{2 \beta+\sigma+1}+(A-2 \beta-1) u^{2 \beta}|\nabla u|^{2}\right] \rho .
$$

In a similar, but easier way, using (3.3) one verifies that

$$
\begin{aligned}
& -\int \varphi^{-1} u^{2 \beta+2}\langle\nabla \varphi, \nabla \rho\rangle \\
& \leq \int\left[-H a(x) u^{2 \beta+2}-(K+1) u^{2 \beta+2} \frac{|\nabla \varphi|^{2}}{\varphi^{2}}+2(\beta+1) u^{2 \beta+1}\left\langle\frac{\nabla \varphi}{\varphi}, \nabla u\right\rangle\right] \rho .
\end{aligned}
$$

Substituting (3.11) and (3.12) into (3.9), recalling the value of $\alpha$, and the condition satisfied by $\beta$, we conclude that

$$
I \leq(\beta+1) \int\left[(A-\beta) u^{2 \beta}|\nabla u|^{2} \rho+\frac{\beta+1-H(K+1)}{H^{2}} u^{2 \beta+2} \frac{|\nabla \varphi|^{2}}{\varphi^{2}} \rho\right] \leq 0,
$$

as required to show that (3.8) holds.

Remark 3.2. Observe that the above proof actually shows that if $A<H(K+1)-1$, then $\nabla u=0$, so that $u$, and therefore $\varphi$, are necessarily constant. It follows from (3.3) and (3.4) that $0 \geq a(x) u \geq b(x) u^{\sigma+1} \geq 0$, so that, if $a$ does not vanish identically, then $u \equiv 0$, without any assumption on $b$. On the other hand, if $A=H(K+1)-1$, then the conclusion depends on the fact that $b$ is positive somewhere.

Remark 3.3. Let $L_{H}$ be the Schrödinger operator defined by $L_{H}=\Delta+H a(x)$. Then the validity of (3.3) is related to the sign of $\lambda_{1}^{L_{H}}(M)$. Indeed, assume that 
$\varphi$ is a positive $C^{2}$ solution of (3.3). Let $\psi \in C_{c}^{\infty}(M)$ and apply the divergence theorem to the vector field $\psi^{2} \nabla \log \varphi$. Since, by (3.3) and Young's inequality,

$$
\begin{aligned}
\operatorname{div}\left(\psi^{2} \nabla \log \varphi\right) & =\frac{\psi^{2}}{\varphi}\left(\Delta \varphi-\frac{|\nabla \varphi|^{2}}{\varphi}\right)+2 \frac{\psi}{\varphi}\langle\nabla \varphi, \nabla \psi\rangle \\
& \leq \frac{\psi^{2}}{\varphi}\left(-H a(x) \varphi-(K+1) \frac{|\nabla \varphi|^{2}}{\varphi}\right)+\frac{\psi^{2}}{\varphi^{2}}|\nabla \varphi|^{2}+|\nabla \psi|^{2},
\end{aligned}
$$

we deduce that

$$
\int_{M}|\nabla \psi|^{2}-H a(x) \psi^{2} \geq K \int_{M} \psi^{2} \frac{|\nabla \varphi|^{2}}{\varphi^{2}},
$$

and from the variational characterization of the bottom of the spectrum, we conclude that if $K \geq 0$, then $\lambda_{1}^{L_{H}}(M) \geq 0$.

On the other hand, if $\lambda_{1}^{L_{H}}(M) \geq 0$, then, by an extension of the result of Moss-Pieperbrink, and Fischer-Colbrie and Schoen quoted in Section 1 (see [PRS3, Lemma 1.2), there exists a positive $C^{1}$ function $v$ which satisfies

$$
\Delta v+H a(x) v=0
$$

weakly on $M$. Further, if $a(x)$ is assumed to be $C^{0, \alpha}$ for some $\alpha \in(0,1)$, then $v$ is $C^{2}$ and it is a classical solution of the above equation. It is clear that $v$ is, respectively, a weak or a classical solution of (3.3) for every $K \leq 0$.

Corollary 3.4. Let $a(x), b(x) \in C^{0}(M)$ and assume that $b(x)$ is non-negative and does not vanish identically. Suppose also that, for some $H \geq 1, \lambda_{1}^{L_{H}}(M) \geq 0$. Then there are no positive $C^{2}$ solutions of the differential inequality

$$
\Delta u+a(x) u-b(x) u^{\sigma} \geq 0, \quad \sigma \in \mathbb{R}
$$

such that

$$
\left(\int_{B_{r}} u^{2(\beta+1)}\right)^{-1} \notin L^{1}(+\infty)
$$

for some $0 \leq \beta \leq H-1$.

Note that, since the corollary deals with strictly positive solutions, we can drop the assumption that $\sigma \geq-1$.

In order to apply Theorem 3.1 and obtain the non-existence result mentioned at the beginning of this section, one needs to verify that the (non-)integrability condition (3.6) holds. In principle, this may be obtained by combining a priori upper estimates for $u$ with appropriate bounds for the volume growth of balls. Both estimates can be deduced by imposing lower bounds on the radial Ricci curvature of the manifold.

In the following lemma we deduce an a priori integral estimate for non-negative solutions of (3.4), which will enable us to obtain (3.6) under the sole assumption of a volume growth condition.

Lemma 3.5. Let $(M,\langle\rangle$,$) be a complete Riemannian manifold, and let a(x), b(x) \in$ $C^{0}(M)$ with $b(x)>0$ on $M$. Assume that $u \geq 0$ is a $C^{2}$ solution of the differential inequality

$$
u \Delta u+a(x) u^{2}-b(x) u^{\sigma+1} \geq-A|\nabla u|^{2},
$$


for $A \leq 1$ and $\sigma>1$. Then for every $p \geq 1, p>A+2$ there exist constants $C_{1}$, $C_{2}>0$ which depend only on $p, \sigma$ and $R_{0}>0$ such that, for every $R \geq R_{0}$,

$$
\int_{B_{R}} b(x) u^{p+\sigma-2} \leq C_{1} R^{-2 \frac{p+\sigma-2}{\sigma-1}} \int_{B_{2 R}} b(x)^{-\frac{p-1}{\sigma-1}}+C_{2} \int_{B_{2 R}}\left(\frac{a_{+}(x)}{b(x)}\right)^{\frac{p-1}{\sigma-1}} a_{+}(x) .
$$

Proof. Observe first that we may assume that $u \not \equiv 0$, for otherwise there is nothing to prove. Thus, there exists $R_{0}>0$ such that $u \neq \equiv 0$ on $B_{R}$ for every $R \geq R_{0}$.

Next, for every $R \geq R_{0}$, let $\psi=\psi_{R}: M \rightarrow[0,1]$ be a smooth cutoff function such that

$$
\psi \equiv 1 \text { on } B_{R}, \quad \psi \equiv 0 \quad \text { on } M \backslash B_{2 R} \text {, and }|\nabla \psi| \leq \frac{C}{R} \psi^{\frac{p-1}{p+\sigma-2}} \quad \text { on } B_{2 R},
$$

for some $C$ which depends only on $p$ and $\sigma$. Note that this is possible since the exponent $\frac{p-1}{p+\sigma-2}$ is strictly less than 1 . Having fixed $\epsilon>0$, we let $W$ be the vector field defined by

$$
W=\psi^{2}(u+\epsilon)^{p-3} u \nabla u .
$$

A computation that uses (3.15) yields

$$
\begin{aligned}
\operatorname{div} W & \geq \psi^{2}(u+\epsilon)^{p-3}\left\{-a(x) u^{2}+b(x) u^{\sigma+1}+\left(1-A-(p-3) \frac{u}{u+\epsilon}\right)|\nabla u|^{2}\right\} \\
& +2 \psi(u+\epsilon)^{p-3} u\langle\nabla u, \nabla \psi\rangle .
\end{aligned}
$$

We estimate the last term on the right hand side by using Cauchy-Schwarz's inequality and Young's inequality $2 a b \leq \lambda a^{2}+\lambda^{-1} b^{2}$ with $\lambda=p-2-A>0$ to obtain

$$
\operatorname{div} W \geq \psi^{2}(u+\epsilon)^{p-3}\left\{-a_{+}(x) u^{2}+b(x) u^{\sigma+1}\right\}-\frac{1}{p-2-A} u(u+\epsilon)^{p-2}|\nabla \psi|^{2} .
$$

We integrate the above inequality, apply the divergence theorem, rearrange, let $\epsilon \rightarrow 0+$ and use the dominated convergence theorem, in this order, to deduce that

$$
\int_{B_{2 R}} b(x) \psi^{2} u^{p+\sigma-2} \leq \frac{1}{p-2-A} \int_{B_{2 R}} u^{p-1}|\nabla \psi|^{2}+\int_{B_{2 R}} \psi^{2} a_{+}(x) u^{p-1} .
$$

If $p=1$ the conclusion follows immediately using 3.17). If $p>1$, we denote by $I$ and $I I$ the two integrals on the right hand side, and use Hölder's inequality with conjugate exponents

$$
\frac{p+\sigma-2}{p-1}(>1) \text { and } \frac{p+\sigma-2}{\sigma-1},
$$

and the assumption that $b(x)>0$ to estimate

$$
I \leq\left(\int_{B_{2 R}} b(x) \psi^{2} u^{p+\sigma-2}\right)^{\frac{p-1}{p+\sigma-2}}\left(\int_{B_{2 R}} \psi^{-2 \frac{p-1}{\sigma-1}} b(x)^{-\frac{p-1}{\sigma-1}}|\nabla \psi|^{2 \frac{p+\sigma-2}{\sigma-1}}\right)^{\frac{\sigma-1}{p+\sigma-2}}
$$

and

$$
I I \leq\left(\int_{B_{2 R}} b(x) \psi^{2} u^{p+\sigma-2}\right)^{\frac{p-1}{p+\sigma-2}}\left(\int_{B_{2 R}} \psi^{2} a_{+}(x)^{\frac{p+\sigma-2}{\sigma-1}} b(x)^{-\frac{p-1}{\sigma-1}}\right)^{\frac{\sigma-1}{p+\sigma-2}} .
$$


Inserting into (3.18), noting that the integral on the left hand side is strictly positive by the choice of $R$, and simplifying, we obtain

$$
\begin{aligned}
\int_{B_{2 R}} b(x) \psi^{2} u^{p+\sigma-2} \leq\left\{\frac{1}{p-2-A}\right. & \left(\int_{B_{2 R}} \psi^{-2 \frac{p-1}{\sigma-1}} b(x)^{-\frac{p-1}{\sigma-1}}|\nabla \psi|^{2 \frac{p+\sigma-2}{\sigma-1}}\right)^{\frac{\sigma-1}{p+\sigma-2}} \\
+ & \left.\left(\int_{B_{2 R}} \psi^{2} a_{+}(x)^{\frac{p+\sigma-2}{\sigma-1}} b(x)^{-\frac{p-1}{\sigma-1}}\right)^{\frac{\sigma-1}{p+\sigma-2}}\right\}^{\frac{p+\sigma-2}{\sigma-1}} .
\end{aligned}
$$

The required conclusion follows again by using (3.17) and the elementary inequality $(a+b)^{\tau} \leq 2^{\tau}\left(a^{\tau}+b^{\tau}\right)$ valid for $a, b, \tau \geq 0$.

We are now ready for our main non-existence result.

Theorem 3.6. Let $(M,\langle\rangle$,$) be a complete Riemannian manifold, and let a(x)$, $b(x) \in C^{0}(M)$, where $b(x)>0$ on $M$ and

$$
b(x) \geq \frac{C}{r(x)^{\mu}}
$$

for $r(x) \gg 1$ and for some constants $C>0$ and $0 \leq \mu \leq 2$. Assume that

$$
\text { (i) } \sup _{M} \frac{a_{+}(x)}{b(x)}<+\infty \text { and (ii) } \int_{B_{r}} a_{+}(x)=O\left(r^{2-\mu} \log r\right) \text { as } r \rightarrow+\infty \text {, }
$$

and that, for some $H \geq 1$, the operator $L_{H}=\Delta+H a(x)$ satisfies

$$
\lambda_{1}^{L_{H}}(M) \geq 0 .
$$

Finally, let $A$ and $\sigma$ be such that $A \leq 1, A<H-1,1<\sigma \leq 2 H+1$ and $\sigma<2 H-A$ and assume that

$$
\operatorname{vol} B_{r}=O\left(r^{2+(2-\mu) \frac{2 H}{\sigma-1}} \log r\right) \text { as } r \rightarrow+\infty .
$$

Then the only non-negative $C^{2}$ solution $u$ of the differential inequality

$$
u \Delta u+a(x) u^{2}-b(x) u^{\sigma+1} \geq-A|\nabla u|^{2}
$$

is $u \equiv 0$.

Proof. If we set $p=2 H+2-\sigma$, the conditions imposed on the parameters imply that $p$ satisfies the assumptions listed in the statement of Lemma 3.5. The lemma and condition (3.20) (i) show that there exist constants $C_{i}>0$ such that

$$
\int_{B_{r}} b(x) u^{2 H} \leq C_{1} r^{-\frac{4 H}{\sigma-1}} \int_{B_{2 r}} b(x)^{1-\frac{2 H}{\sigma-1}}+C_{2} \int_{B_{2 r}} a_{+}(x)
$$

for $r>0$ sufficiently large. We use condition (3.19) to estimate from below the integral on the left hand side. On the other hand, since $\sigma<2 H+1$, we may again use condition (3.19) to estimate from above the first integral on the right hand side, and (3.20) (ii) to estimate from above the second integral, and deduce that, for $r$ sufficiently large,

$$
\int_{B_{r}} u^{2 H} \leq C\left(r^{(\mu-2) \frac{2 H}{\sigma-1}} \operatorname{vol} B_{2 r}+r^{2} \log r\right)
$$

whence, using the volume growth condition (3.22), we conclude that

$$
\int_{B_{r}} u^{2 H} \leq C r^{2} \log r \quad \text { for } r \gg 1
$$


This immediately implies that, for $r$ large,

$$
\frac{r}{\int_{B_{r}} u^{2 H}} \geq C \frac{1}{r \log r} \notin L^{1}(+\infty),
$$

which in turn yields (see, e.g., [RS, Proposition 1.3)

$$
\frac{1}{\int_{\partial B_{r}} u^{2 H}} \notin L^{1}(+\infty) \text {. }
$$

We may therefore apply Theorem 3.1 with $K=0$ and $\beta=H-1$ to deduce that $\operatorname{supp} u=\emptyset$, that is, $u \equiv 0$.

Remark 3.7. The argument used in the proof shows that the condition that $\frac{a_{+}}{b}$ is bounded above may be removed provided we replace (3.20) (ii) with

$$
\int_{B_{r}} a_{+}^{\frac{2 H}{\sigma-1}}=O\left(r^{2-\mu \frac{2 H}{\sigma-1}} \log r\right) \quad \text { as } r \rightarrow+\infty .
$$

Note that since the integral on the left hand side is a non-decreasing function of $r$, this also imposes the further restriction $\mu \leq(\sigma-1) / H$, with corresponding restrictions being imposed on the range of the other parameters.

Remark 3.8. In the case where the ambient manifold is Euclidean space, we can compare our Theorems 3.1 and 3.6 with the results in $\mathrm{AB}$, Section 3. We consider the equation

$$
\Delta u+\lambda a(x) u-u^{2}=0 \quad \text { on } \mathbb{R}^{m},
$$

which, with $a(x)=g(x)$ and a change of scaling, is easily seen to be equivalent to (0.3). We assume, as in $[\mathrm{AB}$, that $a(x)$ is positive somewhere, and that its positive part $a_{+}(x)$ satisfies the estimate

$$
a(x) \leq \frac{k}{|x|^{2}}
$$

for some positive constant $k$. According to the discussion at the end of Section 1, it follows that the principal eigenvalue $\lambda_{*}$ of the linear equation associated to (3.26) is strictly positive and satisfies

$$
\lambda_{*} \geq \frac{(m-2)^{2}}{4 k} .
$$

Moreover, if $\lambda \leq \lambda_{*}$, we have $\lambda_{1}^{\Delta+H \lambda a(x)}\left(\mathbb{R}^{m}\right) \geq 0$ provided $H \leq \frac{\lambda_{*}}{\lambda}$. On the other hand, if $u$ is a non-negative solution of (3.26), then Lemma 3.5 with $A=0, \sigma=2$ and $p>2$ shows that

$$
\int_{B_{r}} u^{p} \leq C \begin{cases}r^{m-2 p} & \text { if } m-2 p>0 \\ \log r & \text { if } m-2 p=0 \\ 1 & \text { if } m-2 p<0\end{cases}
$$

and therefore

$$
\frac{r}{\int_{B_{r}} u^{p}} \notin L^{1}(+\infty)
$$

provided

$$
p \geq \frac{m-2}{2}
$$


In order to apply Corollary 3.4, the non-integrability condition must hold with $p$ satisfying

$$
p=2(\beta+1) \leq 2 H .
$$

Summing up, if $\frac{\lambda_{*}}{\lambda} \geq \min \left\{1, \frac{m-2}{4}\right\}$, then Corollary 3.4 applies, and we conclude that every non-negative solution of (3.26) vanishes identically. To compare with Theorem 3.9 in $\mathrm{AB}$, we point out that we are assuming a less stringent condition on $a_{+}$and that we do not require that $u$ tends to zero at infinity.

On the other hand, assume that $a_{+}(x)$ satisfies the more stringent condition assumed in $\mathrm{AB}$,

$$
a_{+}(x) \leq \min \left\{\frac{k}{|x|^{2}}, \frac{A}{|x|^{2+\delta}}\right\}
$$

for some positive constants $A, k$ and $\delta$. According to [AB], Theorem 3.5, every positive solution of (3.26) which tends to zero at infinity satisfies the estimate

$$
u(x) \leq \frac{C}{|x|^{m-2}},
$$

and, in fact, by Theorem 3.4 therein, every positive solution tends to zero at infinity, provided $a(x)$ is strictly negative off a compact. Now, it is easy to see that if $u$ satisfies (3.28), then

$$
\int_{B_{r}} u^{2} \leq C r^{2}
$$

and, clearly, $\lambda_{1}^{\Delta+\lambda a(x)}\left(\mathbb{R}^{m}\right) \geq 0$ for every $\lambda \leq \lambda_{*}$. An application of Corollary 3.4 with $\beta+1=H=1$ shows that $u$ vanishes identically. We therefore recover the conclusion of Theorem 3.9 in $[\mathrm{AB}$.

Theorem 3.1 does not cover the "endpoint" case where $K=-1$ in (3.3), which we are going to consider presently. We therefore assume that there exists a positive solution $\varphi$ of

$$
\Delta \varphi+H a(x) \varphi \leq \frac{|\nabla \varphi|^{2}}{\varphi}, \quad H>0 .
$$

If $u$ is a $C^{2}$ solution of (3.4) with $\sigma \geq 0$, we define $v=\varphi^{-\gamma} u, \gamma \geq 0$. A computation that uses (3.29), (3.4) and Young's inequality yields

$$
\begin{aligned}
v \Delta v & =(\gamma H-1) a(x) \varphi^{-2 \gamma} u^{2}+b(x) \varphi^{-2 \gamma} u^{\sigma+1} \\
& +\gamma^{2} \varphi^{-2 \gamma-2} u^{2}|\nabla \varphi|^{2}-A \varphi^{-2 \gamma}|\nabla u|^{2}-2 \gamma \varphi^{-2 \gamma-1} u\langle\nabla u, \nabla \varphi\rangle \\
& \geq(\gamma H-1) a(x) \varphi^{-2 \gamma} u^{2}+b(x) \varphi^{-2 \gamma} u^{\sigma+1} \\
& +\gamma^{2}\left(1-\frac{1}{\epsilon}\right) \varphi^{-2 \gamma-2} u^{2}|\nabla \varphi|^{2}-(A+\epsilon) \varphi^{-2 \gamma}|\nabla u|^{2} .
\end{aligned}
$$

Choosing $\gamma=1 / H$ and $\epsilon=-A>0$, the right hand side reduces to

$$
b(x) \varphi^{-2 / H} u^{\sigma+1}+\frac{1}{H^{2}}\left(1+\frac{1}{A}\right) \varphi^{-2 / H-2} u^{2}|\nabla \varphi|^{2},
$$

and we easily deduce that, if $A \leq-1$, then the function $v$ satisfies

$$
\Delta v \geq b(x) \varphi^{(\sigma-1) / H} v^{\sigma} .
$$

Using (3.30) we obtain the following version of Theorem 3.1 . 
Theorem 3.9. Let $a(x), b(x) \in C^{0}(M)$, with $b(x) \geq 0$, and assume that $\varphi$ is a positive $C^{2}$ solution of (3.29) satisfying

$$
\varphi(x) \geq C r(x)^{1 / \delta}
$$

for $r(x) \gg 1$, and some constants $C>0$ and $\delta>0$. Then the differential inequality

$$
u \Delta u+a(x) u^{2}-b(x) u^{\sigma+1} \geq-A|\nabla u|^{2},
$$

with $\sigma \geq 0$ and $A \leq-1$, has no non-negative $C^{2}$ solution satisfying

$$
\operatorname{supp} u \cap\{x: M: b(x)>0\} \neq \emptyset
$$

and

$$
\frac{r^{\delta p}}{\int_{\partial B_{r}} u^{p}} \notin L^{1}(+\infty)
$$

for some $p>1$.

Proof. According to (3.30) above, the function $v=\varphi^{-1 / H} u$ is subharmonic. Further, (3.31) and (3.33) imply that

$$
\left(\int_{\partial B_{r}} v^{p}\right)^{-1} \notin L^{1}(++\infty)
$$

An application of Theorem B in [RS] shows that $v$ is constant. The conclusion now follows as in the proof of Theorem 3.1.

Note that, even in the case of Theorem 3.9 if $A<-1$, then the conclusion can be strengthened to assert that every non-negative solution of (3.9) vanishes identically, unless $a(x)=b(x) \equiv 0$.

In applying Theorem 3.9 it is of course crucial to be able to find positive solutions of (3.29) satisfying the asymptotic lower bound (3.31). By contrast, in order to apply Theorem 3.1 one needs a positive solution of (3.3), whose existence, in typical applications such as the one exemplified by Theorem 3.6 above, is guaranteed by means of assumptions on the spectrum of a suitable operator.

Observe now that if $v$ is a solution of the Poisson equation

$$
\Delta v=a(x),
$$

then the function $\varphi=e^{-v}$ is a positive solution of

$$
\Delta \varphi+a(x) \varphi=\frac{|\nabla \varphi|^{2}}{\varphi}
$$

and furthermore, an upper bound for $v$ yields a lower bound for $\varphi$.

The Poisson equation on complete Riemannian manifolds has been extensively studied using heat kernel techniques to obtain bounds on the Green kernel. To illustrate an application of Theorem 3.9, we consider the elementary case where the positive part of $a(x)$ is integrable. Then we have the following lemma (see, e.g., the proof of Theorem 3.2 in [NST] $)$.

Lemma 3.10. Let $(M,\langle\rangle$,$) be a complete, non-parabolic manifold, and let \rho \in$ $C^{0, \alpha}(M) \cap L^{1}(M)(0 \leq \alpha<1)$ be a non-negative function. Then, there exists a solution $v \in C^{2}$ of the Poisson equation

$$
\Delta v=\rho
$$

satisfying $v \leq 0$. 
Proof. Let $G(x, y)$ be the Green kernel, i.e., the minimal positive fundamental solution of the Laplacian, which exists by the assumption that $M$ is non-parabolic. The Green kernel is symmetric and, if $\psi \in C_{c}^{\infty}(M)$, then the function $u(x)=$ - $\int_{M} G(x, y) \psi(y)$ is smooth and satisfies $\Delta u=\psi$.

We claim that if $\rho \in C^{0}(M) \cap L^{1}(M)$, then the function $v=-\int_{M} G(x, y) \rho(y)$ is well defined and locally bounded. Assuming the claim, for every $\psi \in C_{c}^{\infty}(M)$ we have

$$
\int_{M} v \Delta \psi=-\int_{M} \rho(y) \int_{M} G(x, y) \Delta \psi(x)=\int_{M} \rho(y) \psi(y),
$$

so that $v$ satisfies the Poisson equation in the distributional sense, and therefore, by standard elliptic regularity (see $\mathrm{A}$, Theorem 3.55), it is a classical solution. Clearly, $v$ is non-positive.

To prove the claim, we fix $R>0$ and for every $x \in B_{R}$ we write

$$
\int_{M} G(x, y) \rho(y)=\int_{B_{2 R}} G(x, y) \rho(y)+\int_{M \backslash B_{2 R}} G(x, y) \rho(y) .
$$

Since $G(x, y)$ is locally integrable uniformly for $x \in B_{R}$, the first integral on the right hand side is bounded above by a constant independent of $x \in B_{R}$.

On the other hand, by the local Harnack inequality, there exists a constant $C$ independent of $x \in B_{R}$ and such that

$$
G(x, y) \leq C G(o, y) \quad \text { for every } y \in M \backslash B_{2 R} .
$$

Moreover,

$$
\sup _{M \backslash B_{2 R}} G(o, y)<+\infty .
$$

Indeed, let $\Omega_{n}$ be an exhaustion of $M$ by open sets containing $o$ and with smooth boundary and let $G_{n}$ be the Green kernel of $\Omega_{n}$ and recall that, by the standard construction of the Green kernel $G(x, y), G_{n}(x, y) \rightarrow G(x, y)$ locally uniformly in $M \backslash\{x\}$. Let $C>\sup _{\partial B_{2 R}} G(o, y)$. Then, for every sufficiently large $n$, we have $C>G(o, y) \geq G_{n}(o, y)$ for $y \in \partial B_{2 R}$ and clearly $C>G_{n}(o, y)=0$ for $y \in \partial \Omega_{n}$. Thus, by the comparison principle, $C>G_{n}(o, y)$ in $\Omega \backslash B_{2 R}$, whence, letting $n \rightarrow+\infty, G(o, y) \leq C$ for $y \in M \backslash B_{2 R}$. It follows that there exists a constant $C^{\prime}$ independent of $x \in B_{R}$ and $y \in M \backslash B_{2 R}$ such that

$$
G(x, y) \leq C^{\prime}
$$

Since $\rho$ is integrable, this implies that the second integral on the right hand side of (3.34) is also bounded independently of $x \in B_{R}$, as required to complete the proof of the claim.

Corollary 3.11. Let $(M,\langle\rangle$,$) be a complete, non-parabolic manifold, let the func-$ tions $a(x) \in C^{0, \alpha}(M)$ and $b(x) \in C^{0}(M)$ satisfy $b(x)>0$ and

$$
a_{+}(x) \in L^{1}(M),
$$

and suppose that for some constants $\sigma>1, A \in(-\infty,-1]$ and $\mu, p, q$ satisfying

$$
q>\max \{1,3-\sigma\} \quad 0 \leq \mu \leq 2 \frac{\sigma-1}{\sigma+q-2}, \quad p>\frac{q+\sigma-2}{\sigma-1},
$$


we have

$$
\begin{aligned}
& \int_{B_{r}} a_{+}(x)^{p}=O\left(r^{[2(\sigma-1)-\mu(q+\sigma-2)] \frac{p-1}{q-1}}\right) \text { as } r \rightarrow+\infty, \\
& \operatorname{vol} B_{r}=O\left(r^{2+(2-\mu) \frac{\sigma+q-2}{\sigma-1}}\right) \text { as } r \rightarrow+\infty, \\
& b(x) \geq \frac{C}{r(x)^{\mu}} \text { for } r(x) \gg 1 .
\end{aligned}
$$

Then there are no non-negative, non-identically zero $C^{2}(M)$ solutions of the differential inequality

$$
u \Delta u+a(x) u^{2} \geq b(x) u^{\sigma+1}-A|\nabla u|^{2} \quad \text { on } M .
$$

Proof. Since $a_{+}$is integrable, by Lemma 3.10 and the preceding discussion there exists a solution $\varphi \geq 1$ of

$$
\Delta \varphi+a_{+} \varphi=\frac{|\nabla \varphi|^{2}}{\varphi}
$$

and $\varphi$ is a solution of the differential inequality (3.29).

Now, let $u$ be a non-negative solution of (3.39). Noting that $q>1$, and that $A \leq-1$ implies $q>A+2$, applying Lemma 3.5 and using the lower bound for $b(x)$ (3.38) imply that

$$
\int_{B_{r}} u^{q+\sigma-2} \leq C_{1} r^{(\mu-2) \frac{q+\sigma-2}{\sigma-1}} \operatorname{vol} B_{2 r}+C_{2} r^{\mu \frac{q+\sigma-2}{\sigma-1}} \int_{B_{2 r}} a_{+}(x)^{\frac{\sigma+q-2}{\sigma-1}} .
$$

We claim that (3.36) implies

$$
\int_{B_{2 r}} a_{+}(x)^{\frac{\sigma+q-1}{\sigma-1}}=O\left(r^{2-\mu \frac{\sigma+q-2}{\sigma-1}}\right) \quad \text { as } r \rightarrow+\infty,
$$

which, together with the volume growth assumption (3.37) yields

$$
\int_{B_{r}} u^{q+\sigma-2}=O\left(r^{2}\right) \quad \text { as } r \rightarrow+\infty .
$$

As in the proof of Theorem 3.6, it follows (see, e.g., [RS], Proposition 1.3) that $u$ satisfies condition (3.33) with $\delta=0$ and exponent $q+\sigma-2$, which is greater than 1 by the conditions on $q$. Thus, Theorem 3.9 (with $a(x)$ replaced by $a_{+}(x)$ ) applies and $u$ vanishes identically.

To conclude the proof it remains to prove the claim. To this end, we set $p^{\prime}=(q+$ $\sigma-2) /(\sigma-1)$ and apply Hölder's inequality with conjugate exponents $(p-1) /\left(p-p^{\prime}\right)$ and $(p-1) /\left(p^{\prime}-1\right)$ to estimate

$$
\begin{aligned}
\int_{B_{r}} a_{+}(x)^{p^{\prime}} & =\int_{B_{r}} a_{+}(x)^{\frac{p-p^{\prime}}{p-1}+p \frac{p^{\prime}-1}{p-1}} \\
& \leq\left(\int_{B_{2 r}} a_{+}(x)\right)^{\frac{p-p^{\prime}}{p-1}}\left(\int_{B_{2 r}} a_{+}(x)^{p}\right)^{\frac{p^{\prime}-1}{p-1}} \\
& =O\left(r^{[2(\sigma-1)-\mu(q+\sigma-2)] \frac{p^{\prime}-1}{q-1}}\right)=O\left(r^{2-\mu \frac{q+\sigma-2}{\sigma-1}}\right),
\end{aligned}
$$

as required. 
Remark 3.12. Assume that $b(x)$ satisfies the condition stated in the corollary, with $\mu<\sigma-1$, and that conditions (3.36) and (3.37) are replaced by

$$
\begin{aligned}
& \int_{B_{r}} a_{+}(x)^{\frac{2}{\sigma-1}}=O\left(r^{2\left[1-\frac{\mu}{\sigma-1}\right]}\right) \text { as } r \rightarrow+\infty, \\
& \operatorname{vol} B_{r}=O\left(r^{2\left[1-\frac{\mu-2}{\sigma-1}\right]}\right) \text { as } r \rightarrow+\infty .
\end{aligned}
$$

It follows from (3.40) above with $q+\sigma-2=2$ that every non-negative solution of

$$
\Delta u+a(x) u-b(x) u^{\sigma}=0
$$

satisfies

$$
\int_{B_{r}} u^{2} \leq C r^{2} \log r
$$

and the same estimate is clearly satisfied by the difference of two solutions. An application of Theorem 4.1 in BRS2 shows that (3.42) has at most one positive solution. We remark in this respect that if we replace $u^{2}$ in (3.43) with $u^{p}$ with $p>2$, then the conclusion of Theorem 4.1 in BRS2 fails, as the example described on pages 214-215 therein shows.

\section{REFERENCES}

[A] T. Aubin, Some nonlinear problems in Riemannian geometry. Springer Monographs in Mathematics. Springer-Verlag, Berlin, 1998. MR.1636569 (99i:58001)

[AB] G.A. Afrouzi, K.J. Brown, On a diffusion logistic equation, J. Math. Analysis and Appl. 225 (1998), 326-339. MR 1639260 (99g:35130)

[AW] D.G. Aronson, H.F. Weinberger, Multidimensional nonlinear diffusion arising in population genetics, Advances in Math. 30 (1978), 33-76. MR.511740 (80a:35013)

[BdCS] P. Bérard, M. do Carmo, W. Santos, The index of constant mean curvature surfaces in hyperbolic 3-space, Math. Z. 224 (1997), 313-326. MR1431198 (98a:53008)

[BR] B. Bianchini, M. Rigoli, Nonexistence and uniqueness of positive solutions of Yamabe type equations on nonpositively curved manifolds, Trans. Amer. Math. Soc. 349 (1997), 4753-4774. MR1401514 (98c:53048)

[BRS1] L. Brandolini, M. Rigoli, A.G. Setti, Positive solutions of Yamabe-type equations on the Heisenberg group, Duke Math. J. 91 (1998), 241-296. MR1600582 (99d:35027)

[BRS2] L. Brandolini, M. Rigoli, A.G. Setti, Positive solutions of Yamabe type equations on complete manifolds and applications, Jour. Funct. Anal. 160 (1998), 176-222. MR1658696 (2000a:35051)

[Ch] I. Chavel, Riemannian Geometry - A Modern Introduction. Cambridge University Press, Cambridge, 1993. MR1271141 (95j:53001)

[DM1] Y. Du, L. Ma, Logistic type equations on $\mathbb{R}^{N}$ by a squeezing method involving boundary blow-up solutions, J. London Math. Soc. 64 (2001), 107-124. MR1840774 (2002d:35089)

[DM2] Y. Du, L. Ma, Positive solutions of an elliptic partial differential equation on $\mathbb{R}^{N}$, J. Math. Anal. Appl. 271 (2002) 409-425. MR1923643 (2003f:35106)

[F] H. Federer, Geometric Measure Theory. Springer-Verlag, Berlin, Heidelberg, New York, 1969. MR0257325 (41:1976)

[FCS] D. Fischer-Colbrie, R. Schoen, The structure of complete stable minimal surfaces in 3manifolds of non-negative scalar curvature, Comm. Pure Appl. Math. XXXIII (1980), 199-211. MR.562550 (81i:53044)

[GT] D. Gilbarg, N. Trudinger, Elliptic Partial Differential Equations of Second Order, second edition, Springer-Verlag, Berlin, 1983. MR737190 (86c:35035)

[GW] R.E. Greene, H.H. Wu, Function Theory on Manifolds Which Possess a Pole, Lecture Notes in Mathematics 699, Springer-Verlag, Berlin 1979. MR521983 (81a:53002)

[HK] P. Hess, T. Kato, On some nonlinear eigenvalue problems with an indefinite weight function, Comm. Partial Differential Equations 5 (1980), 999-1030. MR.588690 (81m:35102) 
[J] Z. Jin, Principal eigenvalues with indefinite weight functions, Trans. Amer. Math. Soc. 349 (1997), 1945-1959. MR1389781 (97h:35056)

[K] J.L. Kazdan, Prescribing the Curvature of a Riemannian Manifold, CBMS 57, AMS, Providence, R.I., 1985. MR787227 (86h:53001)

[LTY] P. Li, L.-F. Tam, D. Yang, On the elliptic equation $\Delta u+k u-K u^{p}$ on complete Riemannian manifolds and their geometric applications, Trans. Amer. Math. Soc. 350 (1998), 1045-1078. MR1407497 (98e:58175)

[MM] A. Manes, A.M. Micheletti, Un'estensione della teoria variazionale classica degli autovalori per operatori ellittici del secondo ordine, Bollettino U.M.I. 7 (1973), 285-301. MR0344663 (49:9402)

[MP] W.F. Moss, J. Pieperbrink, Positive solutions of elliptic equations, Pacific J. Math 75 (1978), 219-226. MR500041 (80b:35008)

[NST] L. Ni, Y. Shi, L.F. Tam, Poisson equation, Poincaré-Lelong equation and curvature decay on complete Kähler manifolds, J. Diff. Geom. 57 (2001), 339-388. MR1879230 (2002j:53042)

[PRS1] S. Pigola, M. Rigoli, A.G. Setti, Volume growth, "a priori" estimates, and geometric applications, Geom. Funct. Anal., 13 (2003), 1302-1328. MR2033840 (2004k:53051)

[PRS2] S. Pigola, M. Rigoli, A.G. Setti, Maximum principle on Riemannian manifolds and applications, Memoirs of the AMS 174 no. 822, 2005. MR.2116555 (2006b:53048)

[PRS3] S. Pigola, M. Rigoli, A.G. Setti, Vanishing theorems on Riemannian manifolds and geometric applications, Jour. Funct. Anal. 229 (2005), 424-461. MR.2182595 (2006k:53055)

[PRS4] S. Pigola, M. Rigoli, A.G. Setti, A finiteness theorem for the space of $L^{p}$-harmonic sections, Revista Mat. Iberoamericana 24 (2008), 91-116. MR2435968

[RS] M. Rigoli and A.G. Setti, Liouville-type theorems for $\varphi$-subharmonic functions, Rev. Mat. Iberoamericana, 17 (2001), 471-520. MR 1900893 (2003b:58049)

[Y] S.T. Yau, Some function theoretic properties of complete Riemannian manifold and their applications to geometry, Indiana Univ. Math. J. 25 (1976), 659-670. MR0417452 $(54: 5502)$

Dipartimento di Fisica e Matematica, Università dell'Insubria - Como, via Valleggio 11, I-22100 COMO, ITALY

E-mail address: stefano.pigola@uninsubria.it

Dipartimento di Matematica, Università di Milano, via Saldini 50, I-20133 Milano, ITALY

E-mail address: rigoli@mat.unimi.it

Dipartimento di Fisica e Matematica, Università dell'Insubria - Como, via Valleggio 11, I-22100 Como, Italy

E-mail address: alberto.setti@uninsubria.it 